\title{
The Spin of the Near\#Extreme Kerr Black Hole GRS 1915+105
}

\section{Citation}

McClintock, Jeffrey E., Rebecca Shafee, Ramesh Narayan, Ronald A. Remillard, Shane W. Davis, and Li\#Xin Li. 2006. "The Spin of the Near\#Extreme Kerr Black Hole GRS 1915+105." The Astrophysical Journal 652 (1): 518-39. https://doi.org/10.1086/508457.

\section{Permanent link}

http://nrs.harvard.edu/urn-3:HUL.InstRepos:41384867

\section{Terms of Use}

This article was downloaded from Harvard University's DASH repository, and is made available under the terms and conditions applicable to Other Posted Material, as set forth at http:// nrs.harvard.edu/urn-3:HUL.InstRepos:dash.current.terms-of-use\#LAA

\section{Share Your Story}

The Harvard community has made this article openly available.

Please share how this access benefits you. Submit a story.

Accessibility 


\title{
The Spin of the Near-Extreme Kerr Black Hole GRS 1915+105
}

\author{
Jeffrey E. McClintock ${ }^{1}$, Rebecca Shafee ${ }^{2}$, Ramesh Narayan ${ }^{1}$, Ronald A. Remillard ${ }^{3}$, \\ Shane W. Davis ${ }^{4}$, Li-Xin $\mathrm{Li}^{5}$
}

\begin{abstract}
Based on a spectral analysis of the X-ray continuum that employs a fully relativistic accretion-disk model, we conclude that the compact primary of the binary X-ray source GRS $1915+105$ is a rapidly-rotating Kerr black hole. We find a lower limit on the dimensionless spin parameter of $a_{*}>0.98$. Our result is robust in the sense that it is independent of the details of the data analysis and insensitive to the uncertainties in the mass and distance of the black hole. Furthermore, our accretion-disk model includes an advanced treatment of spectral hardening. Our data selection relies on a rigorous and quantitative definition of the thermal state of black hole binaries, which we used to screen all of the available RXTE and ASCA data for the thermal state of GRS 1915+105. In addition, we focus on those data for which the accretion disk luminosity is less than $30 \%$ of the Eddington luminosity. We argue that these low-luminosity data are most appropriate for the thin $\alpha$-disk model that we employ. We assume that there is zero torque at the inner edge of the disk, as is likely when the disk is thin, although we show that the presence of a significant torque does not affect our results. Our model and the model of the relativistic jets observed for this source constrain the distance and black hole mass and could thus be tested by determining a VLBA parallax distance and improving the measurement of the mass function. Finally, we comment on the significance of our results for relativistic-jet and core-collapse models, and for the detection of gravitational waves.
\end{abstract}

Subject headings: X-ray: stars - accretion, accretion disks — black hole physics — stars: individual (GRS 1915+105)

\footnotetext{
${ }^{1}$ Harvard-Smithsonian Center for Astrophysics, 60 Garden Street, Cambridge, MA 02138

${ }^{2}$ Harvard University, Department of Physics, 17 Oxford Street, Cambridge, MA 02138

${ }^{3}$ Kavli Center for Astrophysics and Space Research, Massachusetts Institute of Technology, Cambridge, MA 02139

${ }^{4}$ Department of Physics, University of California, Santa Barbara, CA 93106

${ }^{5}$ Max-Planck-Institut für Astrophysik, Karl-Schwarzschild-Str. 1, Postfach 1317, 85741 Garching, Germany
} 


\section{Introduction}

GRS 1915+105 has unique and striking properties that sharply distinguish it from the 40 known binaries that are believed to contain a stellar-mass black hole (Remillard \& McClintock 2006, hereafter RM06). It is the most reliable source of highly relativistic radio jets in the Galaxy (Mirabel \& Rodríguez 1994; Fender et al. 1999; Miller-Jones et al. 2006), and it is the prototype of the microquasars (Mirabel \& Rodriguez 1999). GRS 1915+105 (hereafter GRS1915) frequently displays extraordinary X-ray variability that is not mimicked by any other black hole system (e.g., Belloni et al. 2000; Klein-Wolt et al. 2002). Its black hole $(\mathrm{BH})$ primary is unique in displaying a constellation of high-frequency QPOs (HFQPOs), namely, $41 \mathrm{~Hz}, 67 \mathrm{~Hz}, 113 \mathrm{~Hz}$ and $166 \mathrm{~Hz}$. The $67 \mathrm{~Hz}$ QPO is atypically coherent $(Q \equiv \nu / \Delta \nu \sim 20)$ and relatively strong ( $\mathrm{rms}>1 \%$ ) compared to the HFQPOs observed for six other accreting BHs (Morgan et al. 1997; McClintock \& Remillard 2006, hereafter MR06). Among the 17 transient and ephemeral systems that contain a dynamically confirmed BH (RM06), GRS1915 is unique in having remained active for more than a decade since its discovery during outburst in 1992 (MR06). GRS1915 has an orbital period of 33.5 days and is the widest of the $\mathrm{BH}$ binaries (BHBs), and it likely contains the most massive stellar BH (Greiner et al. 2001; Harlaftis \& Greiner 2004; RM06).

Zhang et al. (1997) first argued that the relativistic jets and extraordinary X-ray behavior of GRS1915 are due to the high spin of its BH primary. In their approximate analysis, they found that both GRS1915 and GRO J1655-40 had high spins, $a_{*}>0.9\left(a_{*}=c J / G M^{2}\right.$, where $M$ and $J$ are the mass and angular momentum of the BH; $a_{*}=0$ for a Schwarzschild hole and $a_{*}=1$ for an extreme Kerr hole). Subsequently, Gierlinśki et al. (2001) estimated the spin of GRO J1655-40 and LMC X-3. Recently, we have firmly established the methodology pioneered by Zhang et al. and Gierlinśki et al. by constructing relativistic accretion disk models (Li et al. 2005; Davis et al. 2005) and by modeling in detail the effects of spectral hardening (Davis et al. 2005, 2006). We have made these analysis tools publicly available via XSPEC (kerrbb and bhspec; Arnaud 1996). Using this modern methodology, spins have now been estimated for several stellar-mass BHs, most notably: GRO J1655-40 and 4U 1543-47 (Shafee et al. 2006, hereafter S06), GRS1915 (Middleton et al. 2006), and LMC X-3 (Davis et al. 2006).

All of the plausibly reliable estimates of $\mathrm{BH}$ spin to date, including the present work, depend on fits to the X-ray continuum and measurements of the X-ray luminosity, coupled

with optical measurements of BH mass, orbital inclination, and distance (e.g., S06). In this paper, we show that GRS1915 does indeed harbor a rapidly-spinning Kerr BH as suggested by Zhang et al. (1997). However, in the case of GRO J1655-40 the results obtained by ourselves and others show that the spin of this BH is modest $\left(a_{*} \sim 0.75\right.$; S06; Gierlinśki et 
al. 2001) and much lower than the value $\left(a_{*} \sim 0.93\right)$ suggested by Zhang et al. The high spin reported herein for GRS1915 contradicts the modest spin value $\left(a_{*} \sim 0.7\right)$ reported by Middleton et al. (2006), and we discuss this inconsistency in detail in $§ 5.3$.

Our spin estimates are based on an analysis of the "thermal state" of BHBs (MR06) whose remarkably simple properties have been recognized for decades. Basic principles of physics predict that accreting BHs should radiate thermal emission from the inner accretion disk, and a multi-temperature model of a thin accretion disk was published shortly after the launch of Uhuru (Pringle \& Rees 1972; Shakura \& Sunyaev 1973; Novikov \& Thorne 1973; Lynden-Bell \& Pringle 1974). A nonrelativistic approximation to this model, now referred to as diskbb in XSPEC (Arnaud 1996) was first implemented and used extensively by Mitsuda et al. (1984) and Makishima et al. (1986). The two parameters of the model are the temperature $T_{\text {in }}$ and radius $R_{\text {in }}$ of the inner edge of the accretion disk. In their review on BHBs, Tanaka and Lewin (1995) show for a few BHBs (see their Fig. 3.14) that as the thermal disk flux varies by 1-2 orders of magnitude the value of $R_{\text {in }}$ remains constant to within $\lesssim 20 \%$. This striking result prompted Tanaka \& Lewin to comment that $R_{\text {in }}$, which was typically found to be $\sim$ tens of kilometers, must be related to the radius of the innermost stable circular orbit $\left(R_{\mathrm{ISCO}}\right)$. The stability of $R_{\text {in }}$ has by now been observed in great detail for many BHBs (e.g., Ebisawa et al. 1994; Sobczak et al. 1999; Sobczak et al. 2000; Park et al. 2004). Further strong evidence for a thermal disk interpretation is provided by plots of the observed disk flux versus apparent temperature, which track the expected $L \propto T^{4}$ relation for a constant inner disk radius (Gierlinśki \& Done 2004; Kubota \& Done 2004).

Spin can be determined because it has a profound impact on the behavior and properties of a BH. Quantitatively and specifically, consider two BHs with the same mass $M$, one a Schwarzschild hole and the other an extreme Kerr hole. For the Kerr hole, the radius of the ISCO is six times smaller and the binding energy at the ISCO seven times greater than for the Schwarzschild hole. Relative to the spinless BH, the much deeper gravity well of the extreme Kerr hole hardens the X-ray spectrum and greatly increases its efficiency for converting accreted rest mass into radiant energy. The continuum fitting approach that we use is based on measuring spectral shape (hardness) and luminosity (efficiency).

This paper is organized as follows. In $\S \S 2-4$ we discuss respectively the selection, reduction, and analysis of the data. In $\S 5$ we present our results for GRS1915 and compare them with those of Middleton et al. (2006), and we present a table summary of the spins of GRS1915 and three other BHs. The discussion topics in $\S 6$ include a description of our methodology, our rationale for favoring low-luminosity data, the natal origin of BH spin, the significance of measuring BH spin, and a proposed test of our model. In $\S 7$ we offer our conclusions. 


\section{Data Selection}

Our primary resource is the huge and growing archive of data on GRS1915 that has been obtained during the past decade using the large-area PCA detector on board the Rossi X-ray Timing Explorer (RXTE; Swank 1998). Many BHBs have by now been observed hundreds of times, but none has been observed more often than GRS1915. The net inventory of RXTE pointed observations on this source from 1996 to the present now totals $4.7 \mathrm{Ms}$, which corresponds to 1311 pointed observations each of duration 1-10 ks.

The unique properties of GRS1915 and the great volume of perplexing data present a serious challenge: Is it possible to identify extended periods of time when GRS1915 was in a genuine pacific state dominated by thermal emission, and can one use these data to obtain a reliable estimate of spin? We believe we have answered "yes" to this challenge by using a quantitative definition of the "thermal state" that is based on our exhaustive studies of many BHBs and BH candidates (MR06; RM06). For a discussion of BH states, see MR06 and RM06, and for precise definitions of the three outburst states - including the thermal state - see Table 2 in RM06. For complete overviews on the evolution and energetics of $\mathrm{BH}$ states for six canonical BHBs (i.e., excluding GRS1915), see $§ 5$ in RM06.

In the thermal state (formerly high/soft state and "thermal dominant" state; MR06), which is the only state relevant to this work, the flux is dominated by blackbody-like emission from the inner accretion disk, QPOs are absent or very weak, and the rms variability is also weak. Quantitatively, the thermal state is defined by two timing criteria and one spectral criterion applied over the energy band 2-20 keV (MR06; RM06): (1) QPOs are absent or very weak: amplitude $<0.005 \%$; (2) the power continuum level integrated over $0.1-10 \mathrm{~Hz}$ is $<0.075 \mathrm{rms}$; and (3) the fraction of the emission contributed by the accretion disk component $f_{\mathrm{D}}$ exceeds $75 \%$ of the total emission.

We now turn to describing how we screened the RXTE data archive for GRS1915 and identified 20 observations as belonging strictly to the thermal state. As a starting point, all of these individual PCA observations of GRS1915 that were publicly available as of 2005 January 1 were organized into 640 data segments, where we sometimes combined brief observations that occurred within an interval of several hours. We then screened for temporal variability, and 338 relatively "steady" observations were identified for which the rms fluctuations in the count rate divided by the mean count rate was $<16 \%$ using 1 -s time bins. Next, a hardness ratio $(H R=8.6-18.0 \mathrm{keV} / 5.0-8.6 \mathrm{keV})$ was computed for each of these 338 observations using the scheme of Muno et al. (2001) to normalize the PCA count rates for several epochs with different PCA gain settings. We then selected a gross sample of 85 observations that displayed the softest spectra $(H R<0.30)$. At this point, we strictly applied the three criteria, which define the thermal state. Applying the timing criteria (1) 
and (2) stated above (i.e., QPO amplitude $<0.005 \%$ and rms continuum power $<0.075$ rms), left us with 47 candidate observations. Finally, based on a decomposition of the spectrum into thermal and nonthermal components, which is described in the following section, we obtained our sample of 20 observations that additionally meets criterion (3) given above, namely, that the thermal disk component contributes $f_{\mathrm{D}}>75 \%$ of the total $2-20 \mathrm{keV}$ flux. It is this final sample of 20 strictly thermal-state observations that is the focus of this work. A catalog of the $20 R X T E$ observations, which span a time interval of 7.5 years, is given in Table 1.

Finally, we screened the 11 archival observations of GRS1915 obtained by the Advanced Satellite for Cosmology and Astrophysics (ASCA) and identified two appropriate thermalstate observations. These two observations, which were made on 1994 September 27 and 1999 April 15, are also cataloged in Table 1. In selecting these data we only applied the spectral criterion (number 3) mentioned above and applied it only over the observed bandpass of 1.2-10 keV. The limited count rates (Table 1) did not allow us to exercise the two timing criteria. Because of these limitations, we are somewhat less certain that these observations correspond to the true thermal state than is the case for the RXTE observations.

\section{Data Reduction}

In our spectral analysis of the $R X T E$ data, we only include pulse-height spectra from PCU-2 because it is almost always operating and because fits to the simple power-law (PL) spectrum of the Crab show that this is the best calibrated proportional counter unit (PCU). Data reduction tools from HEASOFT version 5.2 were used to screen the event files and spectra. Data were taken in the "Standard 2 mode," which provides coverage of the PCA bandpass every $16 \mathrm{~s}$. Data from all Xe gas layers of PCU-2 were added to make the spectra. Background spectra were obtained using the tool pcabackest and the latest "bright source"

background model. Background spectra were subtracted from the total spectra using the tool mathpha. Redistribution matrix files and ancillary response files were freshly generated individually for each PCU layer and combined into a single response file using the tool pcarsp. In fitting each of the 20 pulse-height spectra $(\S 4)$, we used response files that were targeted to the time of each GRS1915 observation.

It is well known that fits to PCA spectra of the Crab Nebula reveal residuals as large as $1 \%$, and we therefore added the customary systematic error of $1 \%$ to all PCU energy channels using the tool grppha (e.g., Sobczak et al. 2000). Because large fit-residuals are often found below $3 \mathrm{keV}$, which cannot be accounted for by any plausible spectral feature, and because the spectrum becomes background-dominated and the calibration less certain 
above $25 \mathrm{keV}$, we restricted our spectral analysis to the $3-25 \mathrm{keV}$ band, which is customary for analysis of PCA spectra obtained after the gain change of 1999 March. We used this same 3-25 keV band even for the 14 PCA data sets that were obtained prior to 1999 March.

Table 1. Observations of GRS 1915+105

\begin{tabular}{|c|c|c|c|c|c|}
\hline $\begin{array}{c}\text { Mission } \\
\text { (Detector) }\end{array}$ & $\begin{array}{l}\text { Obs. } \\
\text { No. }\end{array}$ & $\begin{array}{r}\text { Date }(\mathrm{UT})^{a} \\
(\text { yymmdd) }\end{array}$ & MJD & $\begin{array}{c}\text { Exposure } \\
(\mathrm{s})\end{array}$ & $\begin{array}{l}\text { Count Rate } \\
\left.\text { (counts s }^{-1}\right)\end{array}$ \\
\hline$\overline{\mathrm{ASCA}}$ & 1 & 9940927 & 48988.1 & 6019 & 98.3 \\
\hline (GIS2) & 2 & 990415 & 51283.9 & 7153 & 143.3 \\
\hline RXTE & 1 & 960605 & 50239.5 & 10768 & 2197.5 \\
\hline \multirow[t]{19}{*}{ (PCU2) } & 2 & 960607 & 50241.4 & 10960 & 2356.1 \\
\hline & $3^{c}$ & 960703 & 50267.4 & 3424 & 1402.1 \\
\hline & $4^{c}$ & 960703 & 50267.5 & 2944 & 1367.0 \\
\hline & 5 & 970819 & 50679.2 & 2176 & 4953.9 \\
\hline & 6 & 970819 & 50679.3 & 2608 & 4686.1 \\
\hline & 7 & 970819 & 50679.4 & 3328 & 5117.6 \\
\hline & 8 & 970819 & 50679.5 & 1488 & 4941.5 \\
\hline & 9 & 971111 & 50763.2 & 10432 & 4532.1 \\
\hline & 10 & 971209 & 50791.2 & 4544 & 5181.7 \\
\hline & 11 & 971211 & 50793.4 & 2368 & 4284.8 \\
\hline & 12 & 980220 & 50804.9 & 5472 & 5426.6 \\
\hline & 13 & 980220 & 50864.9 & 1520 & 2726.2 \\
\hline & $14^{c}$ & 980329 & 50901.7 & 2768 & 1282.3 \\
\hline & 15 & 991014 & 51465.6 & 5824 & 4976.6 \\
\hline & 16 & 011024 & 52206.6 & 4384 & 4285.4 \\
\hline & $17^{c}$ & 030101 & 52640.4 & 3184 & 1704.0 \\
\hline & 18 & 031029 & 52941.6 & 4128 & 4445.6 \\
\hline & 19 & 031103 & 52946.6 & 2496 & 4594.9 \\
\hline & $20^{c}$ & 031124 & 52967.5 & 4240 & 1675.1 \\
\hline
\end{tabular}

${ }^{a}$ Start time of observation. MJD $=$ JD $-2,400,000.5$.

${ }^{b}$ PCA (full bandwidth): counts $\mathrm{s}^{-1}$ per PCU; $1 \mathrm{Crab}=2500$ cts s $^{-1}$ per PCU.

${ }^{c}$ Key low-luminosity observations (see $§ 4.2 .1 \& \S 6.1$ ).

All PCA count rates for the 20 pulse-height spectra were corrected for dead time. For all normal events (i.e., good events, rejected events and events in the propane layer) we adopted a dead time of $\tau_{\mathrm{N}}=8.83 \mu \mathrm{s}$, and for Very Long Events we adopted $\tau_{\mathrm{VLE} 1}=59 \mu \mathrm{s}$ for setting $=1$ and $\tau_{\mathrm{VLE} 1}=138 \mu \mathrm{s}$ for setting $=2$. The true event rate corrected for dead time 
divided by the observed rate is then $\equiv R_{\text {corr }} / R=1.0-\left(R_{\mathrm{N}} \times \tau_{\mathrm{N}}+R_{\mathrm{VLE} i} \times \tau_{\mathrm{VLE} i}\right)$, where the index $i$ refers to the VLE setting for a given observation. The dead time corrections ranged from 1.016 to 1.080 .

As in S06, we again found it necessary to correct the effective area of the PCA despite a recent official correction (Jahoda et al. 2006), which was made using a nominal and approximate spectrum of the Crab nebula (Zombeck et al. 1990). We have chosen to correct our 3-25 keV fluxes to the most definitive Crab spectrum available, namely, the PL index $(\Gamma=2.10 \pm 0.03)$ and normalization $\left(A=9.7 \pm 1.0 \mathrm{ph} \mathrm{cm}^{-2} \mathrm{~s}^{-1}\right)$ given by Toor \& Seward (1974) and the hydrogen column given by Willingale et al. (2001), which implies a 3.0-25.0 $\mathrm{keV}$ flux of $2.64 \times 10^{-8} \mathrm{erg} \mathrm{s}^{-1} \mathrm{~cm}^{2}$. We consider the old Toor and Seward results more reliable than the current but preliminary results that are summarized in Kirsch et al. (2005).

We made these corrections to the effective area as follows: We selected 25 Crab observations distributed over the 7.5 years spanned by the $20 R X T E$ observations. The Crab pulse-height spectra were corrected for dead time and joined with their response files in the same manner as described above for the GRS1915 spectra. The Crab spectra were fitted over the range 3-25 keV using a simple PL model with the hydrogen column fixed at $N_{\mathrm{H}}=3.45 \times 10^{21} \mathrm{~cm}^{-2}$ (Willingale et al. 2001), and the energy flux was computed over this same interval. The fluxes so computed systematically exceeded the Crab flux quoted above by the factor $1.091 \pm 0.013$ (rms). Therefore, the fluxes we obtained from the analysis of the 20 spectra $(\S 4)$ were all corrected downward by the reciprocal factor 0.917 .

For the two $A S C A$ spectra (Table 1), we analyzed only the data from the GIS2 detector; the calibration of the GIS3 detector, in particular its gain correction, is less certain. We ignored the data from the SIS detectors because GRS1915 is bright and the pileup effects are troublesome (Kotani et al. 2000), which makes the SIS data less suitable for fitting the broad continuum spectrum that is of interest here. Starting with the unscreened ASCA data files obtained from the HEASARC, we followed as closely as possible the data reduction procedures and criteria mentioned in Kotani et al. (2000). The GIS events for each detector were summed within a radius of $6^{\prime}$ centered on the source position, and the response function of the X-ray telescope (Serlemitsos et al. 1995) was applied. Background was not subtracted for this bright source. A gain correction based on the instrumental gold M-edge was applied. A systematic error of $2 \%$ was added to each energy channel to account for calibration uncertainties, and the standard dead time corrections were applied. No correction to the effective area is required because the GIS effective-area calibrations were based on the Toor \& Seward (1974) spectrum of the Crab (Makishima et al. 1996). 


\section{Data Analysis}

All of the data analysis and model fitting was performed using HEASOFT version 5.2 and XSPEC version 12.2 (Arnaud 1996) except for the model bhspec (see below), which requires XSPEC version 11.3. We first consider the most conventional analysis of all 22 data sets (i.e., $20 R X T E$ plus two $A S C A$ ) using the simple multi-temperature disk blackbody model diskbb and then describe three successive analyses of these data sets using our relativistic disk model.

In all the RXTE spectral fits described herein, we fixed the value of the hydrogen column density at $N_{\mathrm{H}}=4.0 \times 10^{22} \mathrm{~cm}^{-2}$. This value is consistent with the values determined from an analysis of the ASCA GIS data for GRS1915 by Ebisawa et al. (1998), who found that $N_{\mathrm{H}}$ was "always within the range $3.5-4.1 \times 10^{22} \mathrm{~cm}^{-2}$," and by ourselves for observations $\# 1$ and \#2, respectively: $N_{\mathrm{H}}=(3.30 \pm 0.04) \times 10^{22} \mathrm{~cm}^{-2}$ and $N_{\mathrm{H}}=(3.75 \pm 0.04) \times 10^{22} \mathrm{~cm}^{-2}$ (§4.1). Our adopted value of $N_{\mathrm{H}}$ is also in reasonable agreement with the BeppoSAX value determined by Feroci et al. (1999), $N_{\mathrm{H}} \sim 5.6 \times 10^{22} \mathrm{~cm}^{-2}$, and with radio and millimeter determinations of the interstellar column, $N_{\mathrm{H}}=(3.5 \pm 0.3) \times 10^{22} \mathrm{~cm}^{-2}$ (Chapuis \& Corbel 2004).

In the following subsections, we discuss in detail the analysis of the $R X T E$ data over the energy range $3-25 \mathrm{keV}$. All of these fits required a nonthermal "tail" component of emission plus two additional weak line and edge components, which are described below.

On the other hand, the $A S C A$ GIS pulse-height spectra, which were analyzed over the energy range $1.2-8 \mathrm{keV}$ required neither a tail component nor the edge components. Apart from these simplifications, the only difference between the analysis of the $A S C A$ data and the $R X T E$ data is that in the former case we allowed $N_{\mathrm{H}}$ to vary freely. Because of the restricted bandpass of $A S C A$ and the limitations associated with screening these data ( $(2)$, we consider the $A S C A$ results somewhat less reliable than the $R X T E$ results, although in the case of GRO J1655-40 we found good agreement between the two, most notably in the case of one simultaneous observation (S06).

\subsection{Nonrelativistic Disk Blackbody plus Simple Power-law Model}

A basic, conventional model consisting of only three principal components, namely, a multi-temperature disk blackbody (diskbb), a simple PL model (power), and interstellar absorption $\left(\right.$ phabs) with $N_{\mathrm{H}}$ fixed at $4.0 \times 10^{22}$ (MR06) consistently gave unacceptably poor fits to the $R X T E$ data. In the usual way, we added two additional components, a Gaussian line gaussian and a broad Fe absorption edge (smedge; e.g., Ebisawa et al. 1994; Sobczak et 
al. 1999, 2000; Park et al. 2004; MR06). In applying the line component, we followed closely the results obtained from high-resolution ASCA SIS observations of GRS1915. Specifically, in a pair of GRS1915 SIS spectra, Kotani et al. (2000) found a complex of several, relativelynarrow absorption features that extend from $\sim 6.4-8.3 \mathrm{keV}$; for both spectra, the equivalent width of the total complex is $E W \approx 0.13 \mathrm{keV}$. Accordingly, given the limited resolution of the PCA $(\approx 18 \%$ at $6 \mathrm{keV})$, we added to our basic model a broad absorption line with a fixed width of $0.5 \mathrm{keV}$, which we bounded to lie between $6.3 \mathrm{keV}$ and $7.5 \mathrm{keV}$. Then, by adding an additional broad Fe absorption component (smedge) with an edge energy restricted to the range $6.9-9.0 \mathrm{keV}$, we were able to obtain good fits to all $20 R X T E$ spectra. We note that Kotani et al. also used a sharp absorption edge component in their model, and we used such a feature in some cases (see $\S 4.2 .4)$.

Using the model described above, we obtained the values of the parameters and fluxes plotted in Figure 1. There are a total of 8 fit parameters: The disk blackbody temperature $T_{\mathrm{in}}$ and its normalization constant $K$, the PL index $\Gamma$ and its normalization constant, the smedge optical depth $\tau_{\mathrm{S}}$ and the smedge edge energy $E_{\mathrm{S}}$, the central energy of the Gaussian absorption line $E_{\mathrm{Fe}}$ and the intensity of the line $N_{\mathrm{Fe}}$. All the fit parameters, except for the PL normalization parameter, are shown in Figure 1. Also shown is the equivalent width $E W$ of the Gaussian line, the $2-20 \mathrm{keV}$ disk and PL fluxes ( $F_{\mathrm{D}}$ and $F_{\mathrm{PL}}$, respectively), and the ratio of these fluxes $f_{\mathrm{D}}$, which is a key quantity used in the selection of these thermal-state data $(\S 2)$.

Finally, we briefly summarize our ASCA GIS2 fit results. For observation \#1 (Table 1), we find $k T_{\text {in }}=1.66 \pm 0.03 \mathrm{keV}, K=126.1 \pm 9.3$ and $\chi_{\nu}^{2}=1.08$ for 98 dof. For observation \#2 we find $k T_{\text {in }}=1.91 \pm 0.03 \mathrm{keV}, K=137.8 \pm 8.1$ and $\chi_{\nu}^{2}=0.94$ for 159 dof. The values of $N_{\mathrm{H}}$ for both observations are quoted above. A Gaussian absorption line with a central energy of $6.85 \pm 0.04 \mathrm{keV}$ and an equivalent width of $0.11 \mathrm{keV}$ was included in the fit to observation \#1, but was not required or included for observation \#2. Neither a smedge component nor a PL or other tail component of emission was included in these $1.2-8.0 \mathrm{keV}$ fits.

\subsection{Relativistic Analysis}

As in S06, we estimate $a_{*}$ by fitting the thermal component of the X-ray continuum using a fully relativistic model of a thin accretion disk around a Kerr BH (Li et al. 2005). The model, which is available in XSPEC under the name kerrbb, includes all relativistic effects, such as frame dragging, Doppler boosting, gravitational redshift, and light bending. It also includes self-irradiation of the disk ("returning radiation") and the effects of limb darkening. A limitation of kerrbb is that one of its three key fit parameters, namely, the 
spectral hardening factor $f$ that relates the color temperature $T$ and the effective temperature $T_{\text {eff }}$ of the disk emission ( $f=\mathrm{T} / T_{\text {eff }}$; Shimura \& Takahara 1995; Merloni et al. 2000) is treated as a constant.

Because of this limitation of kerrbb our work is based on a second, complementary relativistic disk model called bhspec, which has also been implemented in XSPEC (Davis et al. 2005, hereafter D05; Davis et al. 2006, hereafter D06). It does not include the effects of returning radiation, but it provides state-of-the-art capability for computing the spectral hardening factor $f$. The code bhspec is based on non-LTE atmosphere models within an $\alpha$ viscosity prescription (D05; Shakura \& Sunyaev 1973), has just two principal fit parameters (spin and mass accretion rate), and can be used directly to fit for $a_{*}$ (D06). As we now describe, our approach is to combine the functionalities of bhspec and kerrb into a single code that we call kerrbb2.

The use of this hybrid code kerrbb2 marks an important difference in methodology between our earlier work (S06) and the present one. As discussed in S06, kerrbb has three fit parameters - $a_{*}, f$ and the mass accretion rate $\dot{M}$ - only two of which can be determined at one time. In S06, we fitted for $f$ and $\dot{M}$ with $a_{*}$ fixed, and we also computed the Eddingtonscaled luminosity, $l \equiv L / L_{\text {Edd }}\left[L_{\text {Edd }}=1.3 \times 10^{38} M \mathrm{erg} \mathrm{s}^{-1}\right.$ and $L=L\left(a_{*}, \dot{M}\right)$, e.g., Shapiro \& Teukolsky 1984]. We then plotted $f$ versus $l$ and graphically compared the fit results to a model calculation of $f$ versus $l$ performed using bhspec. Finally, by varying the assumed value of $a_{*}$, we determined our estimate of the spin parameter. In the present work, this procedure has been streamlined using kerrbb2, which we now describe.

The code kerrbb2 is a modified version of kerrbb that contains a pair of look-up tables for $f$ corresponding to two values of the viscosity parameter: $\alpha=0.01,0.1$. The entries in the tables were computed using bhspec. The two tables give $f$ versus $l$ for a wide range of the spin parameter, $0<a_{*}<0.9999$. The computations of $f$ versus $l$ were done using the appropriate, corresponding response matrices and energy ranges used in fitting the spectra with kerrbb. Thus, kerrbb and the subroutine/table computed using bhspec now allow us to directly fit for $a_{*}$ and $l \equiv L / L_{\text {Edd }}$ while retaining the special features of kerrbb (e.g., returning radiation). This hybrid code kerrbb2 is used exclusively in all of the data analysis described herein.

In order to estimate the $\mathrm{BH}$ spin by fitting the broadband X-ray spectrum, one must input known values of the mass $M$ of the $\mathrm{BH}$, the distance $D$ to the binary, and the inclination $i$ of the black-hole spin axis, which for GRS1915 we take to be the inclination of the nonprecessing and stable jets (Fender et al. 1999; Dhawan et al. 2000b). For GRS1915, we adopt the following values for these three parameters: $M=14.0 \pm 4.4 M_{\odot}$ (Harlaftis \& Greiner 2004), $D=11.0 \mathrm{kpc}$ and $i=66^{\circ} \pm 2^{\circ}$ with $D<11.2 \pm 0.8 \mathrm{kpc}$ (Fender et al. 1999). In this 
section we use the nominal values of these parameters, and in $§ 5.2$ we examine the effects on $a_{*}$ of allowing these parameters to vary.

In all of the relativistic model fits described below, we used precisely the same ancillary components with the same constraints that we used in our nonrelativistic analysis (§4.1), namely, the $0.5 \mathrm{keV}$-wide Gaussian absorption line and the broad absorption component (smedge). Furthermore, for all of the results presented below, we switched on limb darkening $($ lflag $=1)$ and returning radiation effects $(\mathrm{rflag}=1)$. We set the torque at the inner boundary of the accretion disk to zero, fixed the normalization to 1 (as appropriate when $M, i$, and $D$ are held fixed), allowed the mass accretion rate to vary freely, and fitted directly for the spin parameter $a_{*}$. In the following subsections, we describe our analysis of the $20 R X T E$ and two $A S C A$ spectra using kerrbb2 in which we applied in turn three different models for the tail component, namely, a simple PL model, a thermal Comptonization model, and a simple PL model plus an exponential cutoff at lower energies.

\subsubsection{Relativistic Disk plus Simple Power-law Model}

We now consider our baseline analysis of the 20 RXTE PCA pulse-height spectra using our relativistic disk model kerrbb2 in conjunction with a simple power-law component power. Following precisely the prescription we used in our nonrelativistic analysis (§4.1), we added two additional components, a broad Fe absorption line with a fixed width of $0.5 \mathrm{keV}$ (Kotani et al. 2000) and a broad Fe absorption edge (e.g., Ebisawa et al. 1994). These two conventional and incidental features, which are required in order to obtain a good fit, are subject to exactly the same constraints as before (§4.1). As stated earlier, these fits were done over the energy range $3-25 \mathrm{keV}$, and the column density was fixed to $N_{\mathrm{H}}=4.0 \times 10^{22}$.

As before (§4.1), there are a total of 8 fit parameters, 6 of which are identical to those described previously: the PL index $\Gamma$ and its normalization constant, the smedge optical depth $\tau_{\mathrm{S}}$ and the smedge edge energy $E_{\mathrm{S}}$, and the central energy of the Gaussian absorption line $E_{\mathrm{Fe}}$ and the intensity of the line $N_{\mathrm{Fe}}$. Of course, the two principal fit parameters are now $a_{*}$ and $\dot{M}$ in place of the temperature and disk normalization constant, which are returned by diskbb. The analysis was done for all 20 RXTE observations for both values of the viscosity parameter.

The fit results are summarized in Figure $2(\alpha=0.01)$ and Figure $3(\alpha=0.1)$ in precisely the same format used in displaying the diskbb results in Figure 1. That is, the structure of these figures (e.g., the order of parameters and the ranges over which the parameters are displayed) is identical to the structure of Figure 1, which summarizes the results of our 
nonrelativistic analysis (§4.1). There are two important differences to note between Figures $2 \& 3$ and Figure 1. First, the obvious difference is that $a_{*}$ and $\dot{M}$ are now displayed in place of $T_{\text {in }}$ and $K$. Secondly, in Figures 2 and 3, the value of the disk fraction $f_{\mathrm{D}}$ in the top panel is in the range $f_{\mathrm{D}} \sim 0.9-1.0$. This is generally significantly greater than the corresponding values of $f_{\mathrm{D}}$ shown in Figure 1 , which occasionally dip down to $f_{\mathrm{D}} \approx 0.75$. Thus kerrbb2 is able to accommodate a larger fraction of the total flux than diskbb or, correspondingly, the model for the tail component is less important when fitting with kerrbb2.

The data points for five of the observations in Figures 2 and 3 are enclosed by blue circles. These are the five lowest-luminosity observations $\left(L / L_{\text {Edd }}<0.3\right)$. They are critically important for our determination of the spin of GRS1915, as we explain in $\S 6.1$ and the Appendix. For four of these observations the values of chi-square are relatively high. As we show in $\S 4.2 .4$, the addition of a minor feature to the spectral model allows us to obtain good fits $\left(\chi_{\nu}^{2} \approx 1\right)$ to these four crucial spectra without significantly affecting the values of the two important parameters, $a_{*}$ and $\dot{M}$.

Finally, we briefly summarize our $A S C A$ GIS2 results for the case $\alpha=0.01$. For observation \#1 (Table 1), we find $a_{*}=0.988 \pm 0.003, \dot{M}=(1.40 \pm 0.08) \times 10^{18} \mathrm{~g} \mathrm{~s}^{-1}$, $N_{\mathrm{H}}=(3.39 \pm 0.04) \times 10^{21} \mathrm{~cm}^{-2}$ and $\chi_{\nu}^{2}=1.25$ for 95 dof. For observation \#2 we find $a_{*}=0.957 \pm 0.005, \dot{M}=(3.66 \pm 0.14) \times 10^{18} \mathrm{~g} \mathrm{~s}^{-1}, N_{\mathrm{H}}=(3.99 \pm 0.04) \times 10^{21} \mathrm{~cm}^{-2}$ and $\chi_{\nu}^{2}=0.82$ for 159 dof. A Gaussian absorption line with a central energy of $6.77 \pm 0.05 \mathrm{keV}$ and an equivalent width of $0.21 \mathrm{keV}$ was included in the fit to observation \#1, but was not required or included for observation \#2. No PL or other tail component of emission was included in these $1.2-8.0 \mathrm{keV}$ fits.

\subsubsection{Relativistic Disk plus Comptonization Model}

In the analysis of the $R X T E$ observations described above in $\S 4.1$ and $\S 4.2 .1$, we found that the PL component sometimes makes a modest contribution to the total flux at energies below $\sim 5 \mathrm{keV}$. We question whether this contribution from the PL is physically reasonable, since the PL is believed to be produced by Comptonization of the soft disk photons by a scattering corona. In order to check if this PL flux affects our results, we next fitted the tail component of emission using a more physically-motivated model for which the disk component dominates more strongly below several keV. Namely, we used a thermal Comptonization model (comptt) in place of the simple PL component (Titarchuk 1994; Hua \& Titarchuk 1995). A drawback of comptt is its complexity; it has four principal parameters: the temperature of the soft input photons $T_{0}$, the coronal plasma temperature $T_{\text {cor }}$, the optical depth of the corona $\tau_{\mathrm{C}}$, and a normalization parameter. 
In determining the spin, we considered three fixed values of $T_{0}(\S 5.1)$ that are centered on $2 \mathrm{keV}$, which is the nominal value of the disk temperature determined in $\S 4.1$. As we show in $\$ 5.1$, this choice is completely unimportant. We also considered two values of the coronal temperature, $T_{\text {cor }}=30 \mathrm{keV}$ and $T_{\text {cor }}=50 \mathrm{keV}$, and we found that this choice is also unimportant. For the purposes of the discussion at hand, we adopt the values $T_{0}=2.0 \mathrm{keV}$ and $T_{\text {cor }}=50 \mathrm{keV}$. Thus, we are left with two fit parameters, $\tau_{\mathrm{C}}$ and the normalization constant. When fitting with no constraints on $\tau_{\mathrm{C}}$, we found that the parameter sometimes ran away to unphysically low values $(\lesssim 0.01)$. We therefore set a hard lower bound on the optical depth: $\tau_{\mathrm{C}}>0.4$ (for $T_{\text {cor }}=50 \mathrm{keV}$ ). This bound is based on the values of the photon index determined in $\S 4.1(\Gamma \lesssim 4)$ and a simple calculation that relies on the Zeldovich approximation as described in $\S 7.5$ of Rybicki \& Lightman (1979). Finally, we set comptt's geometry switch to -1 , thereby selecting disk geometry and interpolated values of the $\beta$ parameter. Our results for the fitting parameters and other quantities are summarized in Figure 4 for $\alpha=0.01$ only. The structure of this figure is identical with that of Figure 2 except that $\Gamma$ is replaced by $\tau_{\mathrm{C}}$ and the PL flux $F_{\mathrm{PL}}$ is replaced by the $2-20 \mathrm{keV}$ flux in the comptt component $F_{\mathrm{C}}$.

\subsubsection{Relativistic Disk plus Cutoff Power-law Model}

Modeling the tail component using the thermal Comptonization model is an effective way to check on the effects of PL flux below $\sim 5 \mathrm{keV}(\S 4.2 .2)$. However, this model is quite complex. Therefore, we now consider a simpler model that allows us to cut off the flux at low energy in an ad hoc way, namely, a simple PL model (§4.2.1) that is cutoff at lower energies by an exponential (expabs* power in XSPEC). This model has three parameters, the two standard PL parameters $(\S 4.2 .1)$ plus a cutoff parameter $E_{\mathrm{c}}$. In $\S 5.1$ we consider three plausible choices for the cutoff energy $\left(E_{\mathrm{c}}=8,10 \& 12 \mathrm{keV}\right)$, but for now we consider only the central value, $E_{\mathrm{c}}=10 \mathrm{keV}$. The fit results for this simple model are summarized

in Figure 5, which is strictly identical in structure to Figure 2. The results shown are for $\alpha=0.01$.

\subsubsection{Introduction of a Sharp Absorption Edge}

Five values of chi-square in Figure 2 (observation nos. 3, 4, 12, 14 \& 17) are relatively high, $\chi_{\nu}^{2} \gtrsim 1.5$, and the fit to observation no. 14 is unacceptably high, $\chi_{\nu}^{2}=3.9(44$ dof). Furthermore, these same observations give similarly high values of chi-square for the Comptonization model (Fig. 4) and the cutoff PL model (Fig. 5) as well. These particular 
observations are important because four of them are low-luminosity observations ( $\$ 4.2 .1$, $\S 6.1$, Appendix). In an effort to improve the fits for these five observations, we followed the lead of Kotani et al. (2000; $§ 4.1)$. Specifically, we added to our spectral model a sharp edge feature (edge in XSPEC), which we bounded to lie in the range 8-13 keV, and we then refitted these five PHA spectra. The results are summarized in Figure 6, where the new parameters and fluxes are plotted as red open circles and the small black data points have been copied from Figure 2. Apart from the new fit results, Figure 6 differs from Figure 2 in that it includes a pair of additional panels displaying the parameters of the edge component, $E_{\mathrm{Ed}}$ and $\tau_{\mathrm{Ed}}$. Note in Figure 6 that the optical depth of the edge component is modest, $\tau_{\text {Ed }} \approx 0.2$, and that the addition of this feature significantly reduces the optical depth of the smedge component. Figure 6 contains two important messages. First, with the addition of the edge component all of the five fits are now $\operatorname{good}\left(\chi_{\nu}^{2} \approx 1\right)$. Secondly, the values of $a_{*}$ and $\dot{M}$ are scarcely affected by the inclusion of the sharp edge (see $\S 5.5$, Fig. 6). Finally, we found that the sharp edge gave the same improvements in chi-square and the same degree of stability in the values of $a_{*}$ and $\dot{M}$ as well when applied to the Comptonization $(\S 4.2 .2)$ and cutoff PL models (§4.2.3).

\subsection{Critique of the Different Analysis Approaches}

The disk fraction $f_{\mathrm{D}}$, which is the ratio of the $2-20 \mathrm{keV}$ thermal disk flux to the flux in the tail component (PL, Compton, or cutoff PL) is an important parameter and it is therefore displayed in the top panels in Figures $1-5$. Note that the value of $f_{\mathrm{D}}$ in Figure 1 never dips below 0.75 for any of the 20 observations, which is a principal selection criterion that we used in selecting these data $(\S 2)$ via the nonrelativistic analysis ( $\$ 4.1)$. The typical value is $\approx 90 \%$, although for two observations $f_{\mathrm{D}}$ does fall below $80 \%$. In the case of the relativistic analyses using the PL tail model, the values of $f_{\mathrm{D}}$ are significantly higher with typical values $\gtrsim 95 \%$ and with few values below $90 \%$ (Figures 2, 3 and 5 ). The comptt tail model consistently gives the highest values of $f_{\mathrm{D}}$, which approach $100 \%$. In $\S 4.2 .2$, we expressed some reservations about the simple PL component's contribution to the total flux at low energies. However, as we show in $\S 5$, our results for the PL model agree well with the results obtained for the other two tail models.

A careful comparison of Figures $1-5$ shows that the Gaussian line parameters $\left(E_{\mathrm{Fe}}\right.$, $\left.N_{\mathrm{Fe}}\right)$, the line's equivalent width $(E W)$, and the smedge parameters $\left(E_{\mathrm{S}}\right.$ and $\left.\tau_{S}\right)$ change very little whether the disk is modeled with diskbb or with kerrbb2 and whether the model for the tail component is a simple PL, a Comptonized plasma, or a cutoff PL. This strongly indicates that these ancillary parameters, which are required in order to obtain a good fit, 
are quite unimportant. Furthermore, the Gaussian and smedge components are relatively weak: the Gaussian line has an $E W \approx 0.2 \mathrm{keV}$, comparable to the $\approx 0.13 \mathrm{keV}$ value reported by Kotani et al. (2000), and the optical depth of the smedge component is moderate, $\tau_{\mathrm{S}} \sim 2$ (for comparison, see Ebisawa et al. 1994; Sobczak et al. 1999, 2000; Park et al. 2004).

Finally, if one considers the principal relativistic fit parameters $-a_{*}$ and $\dot{M}$ - plotted in Figures 2-5, one sees that the corresponding values of these parameters from figure to figure are little affected by the choice of model for the tail component (i.e., PL, Compton, or cutoff PL) or by the inclusion of a sharp absorption edge (\$4.2.4, Fig. 6). Thus, we conclude that our results are robust to the details of the analysis - that is, they depend weakly on the line and edge parameters, and they depend weakly as well on the choice of the model for the tail component of emission.

\section{Results}

In this section, we present our results in the form of plots of the dimensionless spin parameter $a_{*}$ versus the dimensionless luminosity $l \equiv L / L_{\text {Ledd }}$. The Eddington-scaled luminosity $l$ is computed from the two kerrbb2 fit parameters $a_{*}$ and $\dot{M}$ and the BH mass $M$ $(\S 4.2)$. In this section we consider in turn the following topics: (1) Our results for the spin of GRS1915; (2) the effects of varying $M, i$ and $D$; (3) a comparison of our results with those of Middleton et al. (2006); (4) the effects of returning radiation and torque; (5) a lower limit on the spin parameter of $a_{*}>0.98$; and (6) a comparison of this limit with the spins of three other sources.

An important point should be mentioned at the outset. The model that we employ to fit the continuum spectrum of GRS1915 is physically consistent only if (i) the accretion disk is in an optically thick thermal state, and (ii) the disk is geometrically thin in the vertical direction. Through the stringent data selection described earlier we have ensured the first requirement, but the second criterion requires a further restriction of the data. In $\S 6.1$ we make use of a Newtonian analysis to estimate the disk thickness, and in the Appendix we describe a fully relativistic analysis. Based on these two analyses, we show that the accretion disk will be thin at all radii, with a height to radius ratio less than 0.1 , only if the accretion luminosity is less than $30 \%$ of the Eddington luminosity. Only five observations with RXTE and one observation with ASCA satisfy this restriction, and we therefore focus most of our attention on these particular data sets (though we present detailed results for all 22 sets). 


\subsection{Spin versus Luminosity for GRS $1915+105$}

All the results given in this subsection assume the nominal values of the opticallydetermined input parameters given in $\S 4.2: M=14.0 M_{\odot}, i=66^{\circ}$, and $D=11.0 \mathrm{kpc}$ (see $\S 4.2$ ). In the following, we show the results of fitting for the spin parameter using three different tail models in turn - simple PL, thermal Comptonization and cutoff PL - in conjunction with our relativistic disk model kerrbb2.

Figure 7 summarizes our fit results $(\S 4.2 .1)$ obtained using our baseline PL tail model (MR06; RM06). The spin parameter is shown plotted versus the Eddington-scaled luminosity l. The results for all $20 R X T E$ and $2 A S C A$ observations (Table 1) are included in this figure. The results are shown for two value of the viscosity parameter, $\alpha=0.01$ and $\alpha=0.1$. All of the analyses reported herein were computed for both values of $\alpha$; however, for low luminosities $(l \lesssim 0.3$ ), which are strongly favored in this work (see $\S 6.1$ and the Appendix), the spin estimates are quite insensitive to the value of $\alpha$ (e.g., Fig. 7), and we therefore generally show results for only $\alpha=0.01$. Error bars are included in Figure 7, although they are generally too small to be apparent.

The principal result of this paper is captured in the set of six lowest-luminosity data points (5 RXTE and $1 A S C A$ ) in Figure 7, namely that the spin-parameter estimate is very nearly unity for $l \lesssim 0.3$. For the group of four data points at intermediate luminosities, $0.3 \lesssim l \lesssim 0.45$, the estimated value of the spin parameter is somewhat depressed, especially for $\alpha=0.1$. At high luminosities, $l \gtrsim 0.65$, the spin estimate is severely depressed and seen to decrease significantly with increasing $l$. As we discuss in $\S 6.1$ and the Appendix, there are good reasons to focus only on those data that correspond to $l<0.3$. We thus conclude that GRS1915 has a spin parameter close to the maximal Kerr value of $a_{*}=1$.

We now consider the effects of replacing the simple PL model for the tail component of emission with a thermal Comptonization model, comptt. As explained in $\S 4.2 .2$, we considered this model because we had reservations about the behavior of the simple PL model at low energies. Additionally, a Comptonization model is more physically motivated and offers a point of comparison with other studies of spin that exclusively use a supplementary Comptonization model (e.g., Middleton et al. 2006, D06). The fitted parameters of this model are displayed in Figure 8. As discussed in $\S 4.2 .2$, in fitting the data using this component, we fixed the thermal temperature of the soft seed photons at three different trial temperatures $-T_{0}=1.5,2.0$ and $2.5 \mathrm{keV}$ - where the central value was determined from our nonrelativistic analysis ( $\S 4.1$; Fig. 1$)$. Figure 8 shows $a_{*}$ versus $l$ for the three values of $T_{0}$ where it is immediately obvious that the results obtained using comptt do not depend on the temperature of the seed photons over the range considered. Error bars are suppressed, but in all cases their extent is less than the height of the plotting symbols. The results, 
which are shown for $\alpha=0.01$, can be seen to be nearly identical to the results obtained using the PL component for $\alpha=0.01$ (Fig. 7). Again, for $l \lesssim 0.3$ we find that $a_{*} \approx 1$ and for intermediate luminosities the spin is slightly depressed $\left(a_{*} \approx 0.98\right)$. As before, the spin drops very significantly at high luminosities $(l \gtrsim 0.65)$.

Next, we consider the results for the cutoff PL model which, like the thermal Comptonization model, contributes negligibly to the flux at low energies. Relative to the Comptonization model, its chief advantage is its greater simplicity, and its disadvantage is its lack of physical motivation (\$4.2). The results are summarized in Figure 9 for $\alpha=0.01$ and for the three values of the break energy mentioned in $\S 4.2 .2$. The error bars, which do not exceed the size of the plotting symbols, are suppressed. As shown in Figure 9, the results are essentially independent of the choice of cutoff energy. Furthermore, the results for the cutoff PL model at both low and intermediate luminosities are nearly identical to the results obtained with the thermal Comptonization model (Fig. 8) and with the simple PL model for $\alpha=0.01$ (Fig. 7).

Finally, in Figure 10 we show superposed the results obtained using all three tail models for $\alpha=0.01$. This figure clearly demonstrates the robustness of our principal result, namely, that the very high spin of GRS1915 does not depend in any significant way on the model used to resolve the relativistic disk component from the faint, adulterating non-disk component. Furthermore, in $\S 4.2$ we have demonstrated that the minor fitting components, the Gaussian line and the smedge, operate the same in all the fits and are therefore incidental to the results that we have obtained for the spin parameter (Figs. 7-10).

\subsection{Effects of Varying $M, i$ and $D$ on the Spin of GRS $1915+105$}

Under the assumption of an intrinsically symmetric jet ejection, Fender et al. (1999) place an upper limit on the distance to GRS $1915+105$ of $D=11.2 \pm 0.8 \mathrm{kpc}$. Further, Fender et al. treat as realistic only distances that are in the range 9-12 kpc, as indicated by the entries in their Table 2. We follow their lead. As shown in their table, the kinematic

jet model associates with each distance a unique value of the jet inclination (e.g., adopting the MERLIN values, $D=11 \mathrm{kpc}$ corresponds to $i=66^{\circ}$ ), which we take as the spin axis of the $\mathrm{BH}$ and the accretion disk ( $\$ 4.2)$. In turn, each value of $i$ is associated with a definite value of the BH mass via the dynamical results for GRS1915 (Greiner et al. 2001; Harlaftis $\&$ Greiner 2004). Thus, we have a correlated triplet of numbers $D, i$ and $M$, which are given in Table 2 for five values of $D$. In Figure 11a, we show the effects of varying $D$ from $11-12.5 \mathrm{kpc}$. The cases $D=9-10 \mathrm{kpc}$ are not shown because these values drive $a_{*}$ toward higher values and we are interested here in highlighting the lowest values of $a_{*}$. Furthermore, 
as discussed in $\S 6.4$, our fit results indicate that the distance to GRS1915 is unlikely to be less than 9-10 kpc. In Figure $11 \mathrm{a}$ and Table 2, we also include $D=12.5 \mathrm{kpc}$ because this extreme distance was adopted by Middleton et al. (2006).

Table 2. Parameters for GRS 1915+105

\begin{tabular}{ccc}
\hline $\begin{array}{c}\text { Distance } \\
(\mathrm{kpc})\end{array}$ & $\begin{array}{c}\text { Inclination }^{a} \\
(\text { degrees })\end{array}$ & $\begin{array}{c}\text { Mass }^{b} \\
\left(M_{\odot}\right)\end{array}$ \\
\hline \hline 9.0 & 61.5 & 15.5 \\
10.0 & 63.9 & 14.6 \\
11.0 & 66.0 & 14.0 \\
12.0 & 67.8 & 13.5 \\
$12.5^{c, d}$ & 68.6 & 13.3 \\
\hline \hline${ }^{a}$ Fender et al. 1999. \\
${ }^{b}$ Based on $P_{\text {orb }}, K_{2}$ and $M_{2}$ from Harliftis \& Greiner 2004. \\
${ }^{c}$ Adopted by Middleton et. al. 2006. \\
${ }^{d}$ Intrinsic jet velocity $>c$.
\end{tabular}

In addition to the uncertainty in the distance, the dynamically-determined value of the BH mass carries its own sizable uncertainty, $M=14.0 \pm 4.4 M_{\odot}$, because the radial velocity amplitude of the secondary is known only to a precision of $11 \%$ (Greiner et al. 2001; Harlaftis $\&$ Greiner 2004). The effects on the spin due to this uncertainty in the mass are shown in Figure $11 b$. As indicated in the figure, the smallest mass, $M=9.6$, gives the lowest values of spin. Limiting our consideration to $L / L_{\mathrm{Edd}}<0.3$ (§6.1, Appendix), Figure $11 a b$ shows that for most allowable distances and masses the spin parameter is nearly unity (see $§ 5.5$ ). Finally, on a separate and incidental matter, we note that our $R X T E$ results are also insensitive to our adopted value of $N_{\mathrm{H}}$ over the full range indicated ( $(4)$ because the absorbing column is already $\sim 85 \%$ transmitting at the PCA's detector threshold energy of $3 \mathrm{keV}$.

\subsection{Comparison with the Results of Middleton et al. for GRS $1915+105$}

For the nominal $11 \mathrm{kpc}$ distance that we adopt (§5.2), Middleton et al. (2006) report a single, moderate value of the spin parameter of $a_{*} \sim 0.8$ (or $a_{*} \sim 0.7$ for their adopted distance of $12.5 \mathrm{kpc}$ ). As we make clear in $\S 5.5$, the M06 value of $a_{*} \sim 0.8$ is very much less than the value we find: $a_{*} \sim 0.98-0.99$. M06 and we used precisely the same publicly-available relativistic accretion disk models (i.e., kerrbb and bhspec). The key difference between the two studies is in the methodology of data selection. M06 used a quite different approach 
that yielded a restricted data sample comprised solely of high-luminosity observations. As we conclude below, our results are in fact in reasonable agreement with Middleton et al. in this high-luminosity regime, which we argue is unreliable for the determination of spin ( $\S 6.1$, Appendix).

Both M06 and we agree completely on the necessity of selecting spectra that minimize the nonthermal component and that are dominated by disk emission. However, M06's methodologies for selecting such spectra were quite different from ours $(\S 2)$. One difference is that M06 based their initial selection on the state classifications defined by Belloni et al. 2000 (see also Belloni et al. 1997, and Belloni 2004), which were devised primarily to study disk-jet coupling via a unified model of X-ray states and radio jets. We, on the other hand, used quantitative state definitions that are centered on physical models of X-ray states (MR05; MR06). These latter state definitions have been applied more widely to many BH binaries including GROJ 1655-40, 4U 1543-47, XTE J1550-564, H 1743-322, XTE J1859+226, and GX 339-4 (MR05; MR06). A second difference is that we screened all the available data and identified 22 observations that are strictly thermal-state data. This yielded a total of $89 \mathrm{ks}$ of RXTE data and $13 \mathrm{ks}$ of $A S C A$ data (Table 1) compared with the much smaller collection of data considered by M06. It is this larger data sample that allowed us to identify several crucial observations at low luminosities $\left(L / L_{\text {Edd }}<0.3\right)$ that are completely absent in M06's data sample.

In the end, M06 fitted jointly three representative 16-s observations in order to determine a single spin estimate with the nominal value of $a_{*}=0.82$ (for $D=11 \mathrm{kpc}$ ). This single spin value and the three corresponding luminosities are indicated in Figure 12 by the three filled triangles, which are connected by a solid line. As shown, these three luminosities range from $L / L_{\mathrm{Edd}}=0.40-1.45$. Note that MR06's low value of spin, $a_{*} \sim 0.82$, is in reasonable accord with the value of $\approx 0.88$ that we find (Fig. 12 ) for a luminosity of $\approx 80 \%$ of $L_{\text {Edd }}$ using the comptt model, which is analogous to the thcomp tail model that M06 used. Furthermore, their somewhat lower spin value, which is an average over a wide range of luminosity, may be largely due to the inclusion of an observation at super-Eddington luminosity $\left(L / L_{\mathrm{Edd}} \approx 1.45\right.$; Fig. 12$)$. Note also that even their mid-luminosity observation with $L / L_{\text {Edd }} \approx 0.85$ (Fig. 12 ) corresponds to the effective Eddington luminosity for thin-disk geometry (§6.1, Appendix). As already mentioned, and discussed in further detail in $\S 6.1$ and the Appendix, the continuum spectral models used by M06 and us are not self-consistent and become progressively less reliable at higher luminosities. In fact, all three 16 -second observations of M06 correspond to $l>0.3$ and are thus in a regime where a number of the physical assumptions which underly the spectral models are likely to break down. 


\subsection{Effects of Returning Radiation and Nonzero Torque at the ISCO}

All of our results include the self-irradiation of the disk as a result of light deflection (assuming that the disk is infinitely thin, see Li et al. 2005), which we refer to as returning radiation. The effects on our results of turning off the returning radiation ( $\mathrm{rflag}=0$ ) is shown in Figure 13. As indicated in the figure, the returning radiation boosts the luminosity of the disk by several percent, but has no significant effect on the spin parameter. The returning radiation feature is not included in bhspec, the relativistic disk model we used to compute tables of the spectral hardening factor, which were incorporated into kerrbb via a subroutine to create kerrbb2 (§4.2). Both kerrbb2 and bhspec can be used directly to determine the principal fit parameters $a_{*}$ and $\dot{M}$. We made a thorough comparison of the fit results obtained using the two models for GRS1915 (and for 4U 1543-47 and GRO J1655-40

as well). For the purposes of this comparison only, we switched the returning radiation off for kerrbb2 ( $\mathrm{rflag}=0)$. We found that the two models gave very comparable results for $a_{*}$ versus $l$.

Throughout this paper we assume that there is no torque acting at the inner edge of the disk. This assumption is in agreement with the classic and current literature on thindisk accretion, which advocates the use of a zero-torque boundary condition (Shakura \& Sunyaev 1973; Novikov \& Thorne 1973; Afshordi \& Paczyński 2003; Li 2003). However, as discussed in $\S 6.1$, a torque may be present near the ISCO, especially in the case of thicker, higher-luminosity disks. Our model kerrbb2 is quite general and capable of handling positive torques of any magnitude with the dimensionless torque parameter $\eta_{\mathrm{T}}$ defined as the ratio of the power generated by the torque to the gravitational binding energy of the accreted gas (Li et al. 2005). As illustrated in Figure 14, the spin parameter decreases with increasing torque. In the presence of sizable torques, the spin parameter of GRS1915 is significantly depressed at high luminosities, but it is scarcely affected at low luminosities.

\subsection{Summary of Results for GRS $1915+105$ and Three Additional Sources}

In Table 3 we summarize the average values of the spin of GRS1915 returned by kerrbb2 for $L / L_{\text {Edd }}<0.3$ that are based on the nominal values of $M, i$ and $D(\S 4.2)$ and on $\alpha=0.01$. The fit results are given for each of the three tail models ( $(4.2)$. The quantities displayed are the Gaussian-weighted mean value of the spin $a_{*}$ and the standard deviation for $N=5$. As indicated by comparing the two lines in the table, the inclusion of a sharp absorption edge in the spectral model (§4.2.4) has a negligible effect on the value of the spin parameter. 
Table 3: Fitted values of spin for $L / L_{\mathrm{Edd}}<0.3$

\begin{tabular}{c|cc|c|c|c|c}
\hline \hline Object & \multicolumn{6}{|c}{ Model } \\
\hline & \multicolumn{2}{|c|}{ Power Law } & \multicolumn{2}{c}{ Comptt } & \multicolumn{2}{c}{ Cutoff Power Law } \\
\hline & mean & st. dev. & mean & st. dev. & mean & st. dev. \\
\hline \hline GRS 1915+105 & 0.998 & 0.001 & 0.997 & 0.001 & 0.997 & 0.001 \\
GRS 1915+105 & 0.998 & 0.001 & 0.995 & 0.002 & 0.996 & 0.001 \\
\hline \hline
\end{tabular}

${ }^{a}$ Sharp absorption edge excluded from the fit; see $\S 4.2 .4$.

${ }^{b}$ Sharp absorption edge included in the fit; see $\S 4.2 .4$.

The formal and precise values of $a_{*}$ in Table 3 for all three models (with and without the edge) are consistent with the physical limit on the Kerr parameter of $a_{*}=0.998$ computed by Thorne (1974). We consider this agreement accidental given the likely uncertainties in the idealized thin-disk model and the model for spectral hardening, the systematic uncertainties in the data, and the uncertainties in $M, i$ and $D$. Nevertheless, the results in Table 3 indicate a very high value for the spin parameter of $a_{*} \gtrsim 0.99$.

We now consider somewhat lower values of spin that cropped up during our analysis. We restrict our discussion to $L / L_{\text {Ledd }}<0.3$ ( $\S 6.1$, Appendix). For example, for the first $A S C A$ observation (Table 1), we find $a_{*}=0.988 \pm 0.003$ for nominal values of $M, i$ and $D$ (§4.2.1). Figure $11 a$ and $11 b$ show respectively the effects of changing $D$ and $M$ on $a_{*}$. Considering distance, the spin is lowest for $D=12.5 \mathrm{kpc}$ : $a_{*}=0.991 \pm 0.006$ (weighted mean for three observations). Considering mass, the spin is lowest for $M=9.6 M_{\odot}$ (single observation with $\left.a_{*}=0.987 \pm 0.001\right)$. Finally, the values of $a_{*}$ are slightly less if one considers the case $\alpha=0.1$ (Fig. 7). Based on these and other considerations and the results in Table 3, we adopt $a_{*}>0.98$ as a lower limit on the spin parameter.

This lower limit of 0.98 for the spin of GRS1915 and our previously estimated values of $a_{*}$ for GRO J1655-40 and 4U 1543-47 (S06) are summarized in Table 4. We obtained very similar estimates of $a_{*}$ for the latter two sources using our revised code kerrbb2 (§4.2), and we will report on this work in a later paper. Also given in Table 4 is an upper limit on $a_{*}$ for LMC X-3 obtained by D06. Here we provide conservative estimates of spin, which are based on the considerations given above for GRS1915 and the full range of variation of $a_{*}$ considered for GRO J1655-40 and 4U 1543-47 in R06. The spin estimates given in Table 4 are our bottom-line results. 
Table 4. Spin estimates for four sources

\begin{tabular}{ccc}
\hline \hline Black Hole & Mission & $a_{*}$ \\
\hline \hline GRS 1915+105 & RXTE/ASCA & $>0.98$ \\
GRO J1655-40 & RXTE/ASCA & $0.65-0.75$ \\
4U 1543-47 & RXTE & $0.75-0.85$ \\
LMC X-3 & RXTE/BeppoSAX & $<0.26^{a}$ \\
\hline \hline
\end{tabular}

${ }^{a}$ Davis et al. 2006.

It is important to emphasize that the spins of GRO J1655-40 and 4U 1543-47, although sizable, are effectively very much less than that of GRS1915, which in turn is significantly less than the theoretical maximum value of $a_{*}=1$. The implications of the extreme spin of GRS1915 are not immediately apparent if one considers the parameter $a_{*}$ alone. Therefore it is instructive to consider such related dimensionless parameters as the radius of the innermost stable circular orbit (ISCO) $\xi$, the binding energy per unit mass at the ISCO $\eta$, and the Keplerian frequency at the ISCO $\omega_{\mathrm{K}}$, which are all monotonic functions of $a_{*}$ (e.g., Shapiro \& Teukolsky 1984). These three quantities are defined and plotted versus $a_{*}$ in Figure 15, which also shows for the four BHs in question the values of these quantities for our nominal estimates of spin. In this approximate comparison, note that both the nominal Keplerian frequency and the binding energy at the ISCO for GRO J1655-40 and 4U 1543-47 are only half the values indicated for GRS1915.

\section{Discussion}

There are four avenues for measuring spin - continuum fitting, high-frequency QPOs, the Fe K line, and polarimetry (RM06). Because spin is such a critical parameter it is important to attempt to measure it by as many of these methods as possible, as this will provide arguably the best possible check on our results. The best current method, continuum fitting, has the drawback that its application requires accurate estimates of $\mathrm{BH}$ mass $M$, disk inclination $i$, and distance $D$. In contrast, observations of HFQPOs require knowledge of only $M$ to provide a spin estimate, and once the correct model is known this method is likely to offer the most reliable measurements of spin. Presently, however, the leading model of HFQPOs, which was initially proposed by Abramowicz \& Kluzniak (2001), does not provide a useful constraint on $a_{*}$ for GRS1915 because of the wide range of possible resonances and the sizable uncertainty in the BH mass (Török et al. 2005). Another HFQPO model, on the other hand, predicts the precise value $a_{*}=0.99616$ for the spin of GRS1915 (Aschenbach 2004). Broadened iron lines do not even require $M$, although knowledge of $i$ 
is useful in order to avoid having to include that parameter in the fit. However, there are serious sources of uncertainty in the model, including the placement of the continuum, the model of the fluorescing source, and the ionization state of the disk (Reynolds \& Nowak 2003). Furthermore, in the case of GRS1915, the line is seldom seen and has provided poor constraints on the models, and no estimate of spin has been given (Martocchia et al. 2002, 2004; Miller et al. 2004). Polarimetry appears promising because the polarization features of BH disk radiation can be affected strongly by GR effects (Lightman \& Shapiro 1976; Connors et al. 1980; Dovčiak et al. 2004). Unfortunately, however, there have been no such observations of BHBs, and there are no mission opportunities on the horizon. In short, the HFQPO and Fe-line methods are not well enough developed to provide dependable results, and the required polarimetry data are not available, whereas the continuum method, despite its limitations, is already delivering results.

The methodology of the continuum-fitting approach is straightforward and transparent. Its foundation is (1) the simplicity of the rigorously defined thermal state $(\S 1 \& \S 2)$, which matches very closely the predictions of the classic thin disk models ( $(1)$, and (2) the vast amount of X-ray spectral data contained in the NASA/GSFC HEASARC archives for missions ranging from Ginga and RXTE to Chandra and XMM-Newton.

In the thin disk model, there is an axisymmetric radiatively-efficient accretion flow in which, for a given $\mathrm{BH}$ mass $M$, mass accretion rate $\dot{M}$ and $\mathrm{BH}$ spin parameter $a_{*}$, one can calculate very accurately the total luminosity of the disk, $L_{\text {disk }}=\eta \dot{M} c^{2}$. The parameter $\eta$, which measures the radiative efficiency of the disk, is a function only of $a_{*}$ (see Fig. 15). We can also calculate precisely the local radiative flux $F_{\text {disk }}(R)$ emitted at radius $R$ by each surface of the disk. Moreover, the accreting gas is optically thick, and the emission is thermal and blackbody-like, making it straightforward to compute the spectrum of the emission. Most importantly, the inner edge of a thin disk is located very close to the innermost stable circular orbit (ISCO) of the $\mathrm{BH}$ spacetime, whose radius $R_{\mathrm{ISCO}}$ (in gravitational units) is a function only of the spin of the $\mathrm{BH}: R_{\mathrm{ISCO}} /\left(G M / c^{2}\right)=\xi\left(a_{*}\right)$, where $\xi\left(a_{*}\right)$ is a monotonically decreasing function of $a_{*}$ (see Fig. 15). Thus, if one measures the radius of the disk inner edge, and if one also has an estimate of the mass $M$ of the $\mathrm{BH}$, then one can immediately obtain $a_{*}$. This is the principle behind our method of estimating BH spin, which was first described by Zhang et al. (1997).

There is one principal difficulty in applying this method. At the high disk temperatures typically found in BHB disks $\left(T_{\text {in }} \sim 10^{7} \mathrm{~K}\right)$, the spectral hardening factor $f(\S 4.2)$ is expected to deviate substantially from unity. It is therefore important to have a reliable estimate of $f$. Until recently, the only estimate available was that from Shimura \& Takahara (1995), whose seminal but limited study was rather approximate. Within the last year, D05 along with 
Davis \& Hubeny (2006) have computed more accurate disk atmosphere models including metal opacities and have obtained reliable estimates of $f$ as a function of the disk luminosity and inclination. The use of a rigorous and modern estimate of $f$ is absolutely essential for the successful application of this method of estimating BH spin, and it is only now that such an estimate has become available. Nevertheless, even at the lower luminosities we favor (§6.1), the vertical structure of real magnetohydrodynamical (MHD) disks may differ in detail from our models (see $\S 3$ of D06 for details). However, preliminary investigations which incorporate the results of MHD simulations suggest only small changes $(\Delta f / f \lesssim 15 \%)$.

\subsection{Rationale for Reliance on Low-Luminosity Data}

In S06, we argued that the method employed in that paper as well as the present paper to estimate BH spin is most reliable at low disk luminosities. The argument has been amplified by D06 (see their §3.1). The main reason to distrust high luminosity data is that the disk is likely to be vertically thick, whereas the model explicitly assumes a thin disk. The detailed general relativistic ray tracing used in kerrbb, kerrbb2 and bhspec assumes a razor-thin disk whose surface is exactly at $z=0$. So long as the disk thickness $H$ is much less than the local disk radius $R$, we expect only small errors to result from the idealized geometry assumed in the model. However, as $H / R$ increases we expect various geometrical effects to creep in. Although it is hard to be quantitative, it is reasonable to think that the errors will become non-negligible once $H / R>0.1$.

Another important assumption in the models is that there is no torque applied at the inner edge of the disk (§5.4). Krolik (1999) and Gammie (1999) argued that magnetic fields would be amplified near the inner edge of the disk, where the gas begins to free-fall into the $\mathrm{BH}$, and that these fields would apply a torque on the disk. The torque will enhance the energy dissipation near the ISCO and lead to a modification in the profile of the disk flux $F(R)$. If this effect is strong enough it will introduce a large error in the $\mathrm{BH}$ spin estimate. Interestingly, Li (2004) finds that, in some cases, a strong magnetic field connecting the disk and the BH may actually move the inner edge of the disk out and cause a reduction in the luminosity. Afshordi \& Paczyński (2003) suggested that the torques are likely to scale as some positive power of $H / R$ and therefore will be unimportant in very thin disks (see their Figs. 17 and 18). This topic is still under debate and is likely to be settled only with detailed models.

To make progress on this question, one approach is to work with the viscous hydrodynamic disk equations, including pressure and radial dynamics (as in Narayan, Kato \& Honma 1997 and Afshordi \& Paczyński 2003), and to calculate the viscous stress at the 
sonic radius and the rate of viscous energy dissipation as a function of radius for various disk thicknesses. Within the limitations of the $\alpha$-viscosity prescription, this will provide a clean answer to whether or not the torque at the inner edge is important for thin disks. Our preliminary analysis appears to support Afshordi \& Paczyński's (2003) assertion that the torque is unimportant for thin disks. A more detailed, and ultimately more rigorous, approach is to carry out 3D numerical MHD simulations of realistic thin disks, including radiative cooling to keep the disk thin. The only work to date involves non-radiative thick disks and is not yet very useful.

Regardless of the current uncertainty over the magnitude of the torque at the disk inner edge, we note that at low luminosities (when the disk is thin, see below) the effects on the spin parameter of even a sizable torque is quite small (see $\S 5.4$ ).

Another effect that becomes important when the disk is vertically thick is radial advection of energy (Abramowicz et al. 1988, 1995; Narayan \& Yi 1994, 1995). The more energy advection there is in the disk, the less energy is radiated to infinity, and the larger is the deviation of $F(R)$ from the idealized thin disk profile assumed in the model. Thus, it is safest to work with disks that have negligible radial advection, i.e., disks with $H / R \ll 1$.

Let us now estimate the disk thickness $H$ and the ratio $H / R$ for a Newtonian thin accretion disk. The flux emitted by a thin accretion disk around a $\mathrm{BH}$ with zero torque at the inner edge is given by (e.g., Frank et al. 2002)

$$
F(R)=\frac{3 G M \dot{M}}{8 \pi R^{3}}\left[1-\left(\frac{R_{\text {in }}}{R}\right)^{1 / 2}\right]
$$

where $M$ is the mass of the $\mathrm{BH}, \dot{M}$ is the mass accretion rate, $R$ is the cylindrical radius, and $R_{\text {in }}$ is the radius of the inner edge of the disk. Let us define the Eddington mass accretion rate by equating the disk luminosity to the Eddington luminosity,

$$
\frac{G M \dot{M}_{\mathrm{Edd}}}{2 R_{\text {in }}}=L_{\mathrm{Edd}} \equiv \frac{4 \pi G M c}{\kappa}, \quad \text { i.e., } \quad \dot{M}_{\mathrm{Edd}}=\frac{8 \pi c R_{\mathrm{in}}}{\kappa},
$$

where $\kappa$ is the opacity of the gas. Correspondingly, let us define the Eddington-scaled mass accretion rate by

$$
\dot{m} \equiv \frac{\dot{M}}{\dot{M}_{\mathrm{Edd}}}
$$

We now rewrite the disk flux $F(R)$ in terms of $\dot{m}$ and calculate the vertical acceleration due to radiation pressure,

$$
g_{\mathrm{rad}}(R)=\frac{F(R) \kappa}{c}=\frac{3 G M \dot{m}}{R^{2}}\left[\left(\frac{R_{\mathrm{in}}}{R}\right)-\left(\frac{R_{\mathrm{in}}}{R}\right)^{3 / 2}\right] .
$$


In equilibrium, the radiative acceleration must be balanced by the vertical component of gravity, which for simplicity we can write as $g_{z}(R, z)=G M z / R^{3}$. We then find

$$
\frac{H}{R} \approx 3 \dot{m}\left[\left(\frac{R_{\mathrm{in}}}{R}\right)-\left(\frac{R_{\mathrm{in}}}{R}\right)^{3 / 2}\right] .
$$

Figure 16 shows $H / R$ as a function of $R$ for various choices of the accretion rate: from below, $\dot{m}=0.1,0.2, \ldots, 1.2$. If we wish to have $H / R<0.1$ at all $R$, then we see that we are limited to $\dot{m} \lesssim 0.25$, i.e., to Eddington-scaled disk luminosities $l \lesssim 0.25$. The Appendix describes a more accurate estimate of the disk height that is calculated for a general relativistic disk around a Kerr BH. Results are shown in Figure 17. According to that analysis, in order to have $H / R \lesssim 0.1$ at all radii, we must restrict our attention to luminosities $l \lesssim 0.3$. This is the limit we employ throughout the paper.

If we consider the exact expression for the Newtonian vertical gravity

$$
g_{z}(R, z)=\frac{G M z}{\left(R^{2}+z^{2}\right)^{3 / 2}},
$$

rather than the approximation $G M z / R^{3}$, then we find that the maximum value of the vertical gravitational acceleration (which is achieved at $z=R / \sqrt{2}$ ) is

$$
\left(g_{z}\right)_{\max }=\frac{2}{3 \sqrt{3}} \frac{G M}{R^{2}} .
$$

For any accretion rate greater than about $85 \%$ of Eddington, or $\log \left(L / L_{\mathrm{Edd}}\right)>-0.06$, one finds that some parts of the disk produce too much radiation to be balanced even by the maximum vertical gravity $\left(g_{z}\right)_{\max }$. Radiation pressure will then cause material to be blown away from the disk. This critical luminosity is clearly related to the Eddington limit; the slightly different numerical value, i.e., $85 \%$ instead of $100 \%$ of the canonical Eddington limit, is the result of the different geometry of a disk compared to the spherical geometry that one usually considers. (See Nityananda \& Narayan 1982 for a discussion of geometry effects on the Eddington limit.)

We showed in $\S 5$ that, by focusing on data corresponding to $L / L_{\text {Edd }} \lesssim 0.3$, we obtain very consistent results for the spin parameter of GRS1915, independent of the details of the spectral model we employ. We also found that the results begin to deviate as we go to higher luminosities, suggesting that as the disk thickens one or more of the effects described in this subsection becomes important. It is interesting that the deviations are not random but very systematic, e.g., the estimate of $a_{*}$ decreases smoothly and monotonically as $L / L_{\text {Edd }}$ increases. This signature could conceivably be used to identify which of our assumptions breaks down as the luminosity increases. Detailed viscous disk models with varying disk thickness might be able to shed some light on this issue. 


\subsection{The Spins of Stellar Black Holes are Chiefly Natal}

King and Kolb (1999) provide a global evolutionary and observational argument that neither significant spinup nor spindown is likely to occur during the lifetime of any $\mathrm{BH}$ binary and hence that $\mathrm{BH}$ primaries essentially retain the spin rates that they had at birth. In the particular case of $4 \mathrm{U}$ 1543-47, based on its present accretion rate and modest age $(\lesssim 1$ Gyr), we argued that the spin of its BH (§5.7) is likewise chiefly natal (S06). The fast spin reported herein for GRS1915, $a_{*}>0.98$, is almost certainly a natal spin because the alternative, achieving this spin gradually via accretion torques, would require almost doubling the mass of the $\mathrm{BH}$ (see below). Such a large increment in BH mass is unlikely to have occurred during the evolution of GRS1915 or any BH binary simply because systems with initially low- or moderate-mass secondaries (i.e., $M \lesssim$ a few $M_{\odot}$ ) obviously cannot supply the required mass, and systems with high-mass secondaries have lifetimes that are too short to effect the required mass transfer. We now consider the exceptional case of GRS1915 in more detail.

GRS1915 presently has a low-mass secondary, $M_{2}=0.81 \pm 0.53 M_{\odot}$ (Harliftis \& Greiner 2004 ) and the most massive primary and longest period of any BH binary $(\S 1)$. There is a great deal of uncertainty in evolutionary models for GRS1915 and for all BH binaries. The specific evolutionary model of Belczynski \& Bulik (2002) for GRS1915 argues for a small transfer of mass to the primary and negligible spin up, which is in agreement with most generic models (e.g., King \& Kolb 1999). An evolutionary model of GRS1915 that links this source to the ultraluminous X-ray sources implies the most extreme mass transfer and spin up (Podsiadlowski et al. 2003). These authors argue that the initial secondary mass could have been as high as $6 M_{\odot}$ and the BH primary could have accreted as much as $\sim 4 M_{\odot}$ (see also Lee et al. 2002). Even for this extreme scenario, the predicted spin up due to accretion torques is modest. Based on a precise calculation that ignores returning radiation ( $\$ 5.5)$, we find that the transfer of $4 M_{\odot}$ onto a $10 M_{\odot}$ natal black hole with zero initial spin yields a final spin of only $\sim 0.77$, which is far less than our limit of $a_{*}>0.98$ ( $\S 5.5$, Fig. 15). Likewise, to

achieve a final spin of $a_{*}>0.98$ would require an initial spin of $a_{*}>0.75$. Furthermore, if one includes the effects of returning radiation, then the accretion is less efficient in spinning up the hole and a somewhat larger natal spin is required. Again neglecting returning radiation, a $10 M_{\odot} \mathrm{BH}$ that is spun up by accretion torques from $a_{*}=0$ to $a_{*}=0.98$ would have a final mass of $19.3 M_{\odot}$; since some of the rest mass energy is radiated away, the total rest mass accreted in such a spin up event would be $10.7 M_{\odot}$. We thus conclude that the extreme spin of GRS1915 was likely imparted to the BH primary during the process of its formation.

The generation of large spins is central to GRB models. Natal spins of $a_{*} \sim 0.8$, in agreement with our observations, were predicted for GRO J1655-40 and 4U 1543-47 by 
Lee et al. (2002). The extreme spin of GRS1915, $a_{*}>0.98$, is an expected consequence of collapsar models $(\S 6.3)$.

\subsection{Significance of Measuring Black Hole Spin}

The properties of a $\mathrm{BH}$ are completely defined by specifying just two parameters, its mass $M$ and its dimensionless spin parameter $a_{*}$. Furthermore, a BH's mass simply supplies a physical scale, whereas its spin fundamentally changes the geometry of space-time. Accordingly, in order to model the ways in which an accreting BH can interact with its environment, one must know its spin. For example, consider one of the most intriguing unsolved problems in astrophysics, namely, the connection between $\mathrm{BH}$ spin and relativistic jets that are commonly observed for both supermassive and stellar-mass BHs and that are so prominent in the case of GRS1915 (e.g., Mirabel \& Rodríguez 1999). For many years, scientists have speculated that these jets are powered by BH spin via a Penrose-like process associated with magnetic fields (e.g., Blandford \& Znajek 1977; Hawley \& Balbus 2002; Meier 2003; McKinney \& Gammie 2004). However, these ideas will remain mere speculation until sufficient data on BH spins have been amassed and the models can be tested and confirmed. This provides strong motivation for measuring the spins of accreting BHs.

The strong evidence for natal spins - particularly in the case of GRS1915 $(\S 6.2)$ - is obviously of major significance in building core-collapse models for SN and GRBs (Woosley 1993; MacFadyen \& Woosley 1999; Woosley \& Heger 2006). For example, one of the greatest uncertainties in GRB modeling is whether one can arrive at the core collapse stage with sufficient angular momentum to make a disk around a BH. The spins of GRO J1655-40 and 4U 1543-47 - and especially GRS1915 - provide strong evidence for the high natal rotation rates of BHs and thus provide strong support for the collapsar model of "long-soft" GRBs.

The continuing development of gravitational wave astronomy is central to the exploration of $\mathrm{BHs}$, and knowledge of $\mathrm{BH}$ spin is fundamentally important to this effort. To detect the faint coalescence signal for two inspiralling BHs, one must compute the expected waveform and use it to filter the data. Our spin estimates for GRO J1655-40 and 4U 1543-47 (R06) motivated the first such waveform computation that includes the effects of spin (Campanelli et al. 2006), and our results reported here for GRS1915 present a further challenge to the waveform modelers. 


\subsection{An Observational Test of the Spin and Jet Models for GRS $1915+105$}

As mentioned in $§ 5.2$, our fit results for the five low-luminosity observations $(l<0.3$; see $\S 4.2 .1$, Table 1) indicate that the distance to GRS1915 is unlikely to be less than about 9-10 kpc. This result is based on the abrupt and dramatic rise in $\chi^{2}$ that occurs for lesser distances. For the nominal $14.0 M_{\odot}$ value of $\mathrm{BH}$ mass and $D=11.0,10.5,10.0,9.5$ and $9.0 \mathrm{kpc}$, the respective values of $\chi_{\nu}^{2}$ for each low-luminosity observation are 0.6, 0.6, 2.6, 13.3, 43.4 (obs. no. 3); 0.5, 1.4, 2.7, 14.2, 43.8 (obs. no. 4); 0.6, 2.0, 6.3, 23.1, 263.6; (obs. no. 14); 1.0, 1.1, 5.6, 26.8, 77.1 (obs. no. 17); and 0.7, 0.7, 1.4, 12.1, 45.4 (obs. no. 20). This abrupt rise in $\chi^{2}$ indicates that we have reached the limit of our table model $\left(a_{*}=0.9999\right)$ and that the fit is demanding unphysical values of $a_{*}>1$. In Figure 18a, this distance lower limit, which is a function of BH mass, is indicated by the long slant line labeled "spin

model." For each assumed value of mass, and hence inclination and distance (see §5.2), the limiting value plotted in Figure $18 a$ is an average result for the five low-luminosity points at a $99 \%$ level of confidence $\left(\Delta \chi^{2}=6.6\right.$ ). (The results are very insensitive to the binary mass ratio, which we have held fixed at its nominal observed value; Harlaftis \& Greiner 2004.) To the right of the vertical line labeled "jet model," the intrinsic velocity of the radio jet exceeds the velocity of light ( $\$ 5.2$; Fender et al. 1999). The region below the nearly horizontal line is disallowed by the jet model and the $1-\sigma$ lower limit on the mass function $(\$ 5.2$; Greiner et al. 2001). Thus, taken together, the spin and jet models plus the value of the mass function predict that the distance and BH mass of GRS1915 lie within the triangular region shown in the figure.

Six model-dependent estimates of the distance to GRS1915 are summarized in Figure 18b. Some estimates disagree, others are very uncertain, and none provides a convincing test of the constraints summarized in Figure 18a. We believe that it should be possible to obtain a model-independent VLBA parallax distance that is precise to $\sim 10 \%$ and to reduce the uncertainty by a factor of two in the radial velocity amplitude $K$ of the secondary, which would significantly improve the accuracy of the mass function. Such improvements in the observational constraints will provide a powerful test of the spin and jet models for GRS1915.

\section{Conclusions}

Using a rigorous and quantitative definition of the thermal state of a black hole binary (§2), we screened all the available RXTE PCA and ASCA GIS data and identified a total of 22 observations of GRS 1915+105 that are free of QPOs and strong timing noise and for which the thermal disk component of emission contributes $>75 \%$ of the total $2-20 \mathrm{keV}$ flux. We then fitted the 22 disk-dominated spectra using principally a model of a thin accretion disk in 
the Kerr metric that includes all relativistic effects plus an advanced treatment of the spectral hardening factor $f(\S 4.2)$. The spectral fitting of the 22 spectra was repeated a number of times using three different models for the nonthermal tail component of emission and two different values of the viscosity parameter $(\S 4)$. The results for the key relativistic parameters - the spin $a_{*}$ and the mass accretion rate $\dot{M}$ - were shown to be quite independent of any details of the analysis and insensitive to the uncertainties in the independently-determined input parameters, namely, the mass, inclination and distance of the black hole (§5).

On theoretical grounds, we argue that the spin parameter can be determined most reliably at lower luminosities ( $\$ 6.1$, Appendix). Our relativistic disk model assumes a disk that is thin and torque-free at its inner edge. Higher luminosities are problematic because they likely lead to disk thickening and nonzero torques near the ISCO. Based on theoretical arguments, we propose a limit on the disk thickness and a corresponding limit on the disk luminosity, $L / L_{\text {Edd }}<0.3$, below which one can obtain reliable estimates of the spin parameter. Adopting this criterion, we obtain our principal conclusion: GRS 1915+105 is a rapidly-rotating $\mathrm{BH}$ with a lower limit on its spin parameter of $a_{*}>0.98$. Finally, we propose an observational test of our spin model.

We thank Keith Arnaud for help in implementing models in XSPEC and the following people for helpful discussions and encouragement: Stan Woosley, Alexandar Heger, Gerry Brown, Vicky Kalogera, Cole Miller, and Paul Gorenstein. We also thank an anonymous referee for helpful comments and a thorough reading of our paper. This research has made use of data obtained from the High Energy Astrophysics Science Archive Research Center (HEASARC), provided by NASA's Goddard Space Flight Center. This work was supported in part by NASA grant NNG 05GB31G and NSF grant AST 0307433.

\section{A. Vertical Thickness of a Thin Accretion Disk around a Kerr Black Hole}

Following Page \& Thorne (1974), we define the following functions for later use,

$$
\begin{aligned}
& \mathcal{A}=1+a_{*}^{2} x^{-4}+2 a_{*}^{2} x^{-6}, \quad \mathcal{B}=1+a_{*} x^{-3}, \\
& \mathcal{C}=1-3 x^{-2}+2 a_{*} x^{-3}, \quad \mathcal{D}=1-2 x^{-2}+a_{*}^{2} x^{-4} \text {, }
\end{aligned}
$$

where $a_{*} \equiv a / c R_{g}$ is the dimensionless spin of the black hole $\mathrm{BH}, R_{g} \equiv G M / c^{2}$ is the gravitational radius of the $\mathrm{BH}$ of mass $M$, and $x \equiv\left(R / R_{g}\right)^{1 / 2}$. Note, $\mathcal{D}$ vanishes on the horizon of the $\mathrm{BH}$.

On the equatorial plane of the $\mathrm{BH}$, the lapse function and the angular velocity of frame 
dragging are

$$
\chi=\left(\frac{\mathcal{D}}{\mathcal{A}}\right)^{1 / 2}, \quad \omega=\frac{2 a_{*} R_{g}^{2} c}{R^{3} \mathcal{A}} .
$$

The angular velocity of a thin Keplerian disk at radius $R$ is

$$
\Omega_{\mathrm{D}}=\left(\frac{G M}{R^{3}}\right)^{1 / 2} \frac{1}{\mathcal{B}},
$$

and the rotational 3-velocity of the disk relative to the locally nonrotating frame is

$$
v_{\phi}=\frac{\mathcal{A}^{1 / 2}}{\chi}\left(\Omega_{\mathrm{D}}-\omega\right) R .
$$

The 4-velocity of the disk particle is then

$$
U^{a}=\frac{\Gamma}{\chi}\left[\left(\frac{\partial}{\partial t}\right)^{a}+\Omega_{\mathrm{D}}\left(\frac{\partial}{\partial \phi}\right)^{a}\right],
$$

where $\Gamma=\left(1-v_{\phi}^{2} / c^{2}\right)^{-1 / 2}=\mathcal{B} / \mathcal{C}^{1 / 2}$ is the Lorentz factor. The 4-velocity satisfies the normalization condition $U^{a} U_{a}=-1$.

The relative acceleration between two neighboring particles moving on geodesics with a small separation vector $X^{a}$ is given by the geodesic deviation equation (Wald 1984),

$$
g^{a}=-R_{c b d}^{a} X^{b} U^{c} U^{d}
$$

where $R_{c b d}{ }^{a}$ is the Riemann tensor of the spacetime and $U^{a}$ is the four-velocity of the geodesic. The acceleration is measured in the rest frame of the particles. For a particle above the equatorial plane at a small height $z$ and corotating with the disk, we have $X^{a}=z e_{z}^{a}$, where $e_{z}^{a}$ is a normalized unit vector orthogonal to the equatorial plane. Combining this with the $U^{a}$ given in equation (A6) and the Riemann tensor of the Kerr spacetime, we can calculate the relative acceleration,

$$
g^{a}=-g_{z} e_{z}^{a}, \quad g_{z}=\xi \frac{G M z}{R^{3}}
$$

where $^{1}$

$$
\xi=\frac{1}{\mathcal{C}}\left(1-4 a_{*} x^{-3}+3 a_{*}^{2} x^{-4}\right)
$$

\footnotetext{
${ }^{1}$ Our result differs from eq. (5.7.2) of Novikov \& Thorne (1973). After intensive examination, we believe that their formula is incorrect.
} 
For a disk that is radiation-dominated (at least at the photosphere), the equilibrium in the vertical direction is determined by

$$
\left.\frac{F \kappa}{c} \approx g_{z}\right|_{z=H}
$$

where $F=F(R)$ is the radiation flux density of the disk (measured by an observer corotating with the disk) and $\kappa$ is the disk opacity. The flux density $F$ has been derived by Page \& Thorne (1974) and is given by

$$
F=\frac{3 G M \dot{M}}{8 \pi R^{3}} f_{0}
$$

where $f_{0}=\left(2 R^{2} / 3 R_{g}\right) f$ and the expression for $f$ is given in equation (15n) of Page \& Thorne (1974). Our choice of $f_{0}$ instead of $f$ is based on the fact that, unlike $f, f_{0}$ is dimensionless. Note that at $R=R_{\mathrm{ISCO}}$ (the innermost stable circular orbit) we have $f_{0}=0$, and that as $R \rightarrow \infty$ we have $f_{0} \rightarrow 1$.

By equations (A8)-(A10), the scale-height of the disk is

$$
\frac{H}{R} \approx \frac{3 \kappa \dot{M}}{8 \pi R c} \frac{f_{0}}{\xi} .
$$

Following the analysis of the Newtonian case ( $\S 6.1$ ), we define the Eddington luminosity by

$$
L_{\mathrm{Edd}}=\frac{4 \pi G M c}{\kappa}=\varepsilon \dot{M}_{\mathrm{Edd}} c^{2}, \quad \text { i.e., } \quad \dot{M}_{\mathrm{Edd}}=\frac{4 \pi c R_{g}}{\kappa \varepsilon},
$$

except that here $\varepsilon=\varepsilon\left(a_{*}\right)$ is the radiative efficiency of the relativistic disk (see Page \& Thorne 1974). With the above definition of $\dot{M}_{\text {Edd }}$, we have $L / L_{\text {Edd }}=\dot{M} / \dot{M}_{\text {Edd }} \equiv \dot{m}$, where $L$ is the luminosity of the disk. Then, equation (A12) can be recast into

$$
\frac{H}{R} \approx \frac{3 \dot{m}}{2 \varepsilon} \frac{f_{0}}{x^{2} \xi}
$$

It can be shown that this expression for $H / R$ simplifies to equation (5) in the Newtonian limit. Note that $H / R$ does not depend on the value of the opacity $\kappa$.

Since the ratio $H / R=0$ at $R=R_{\mathrm{ISCO}}$ and also as $R \rightarrow \infty$, it must have a maximum at some finite $R>R_{\mathrm{ISCO}}$. It turns out that for any given value of $\dot{m}$, the maximum value of $H / R$ is very insensitive to variation in $a_{*}$ (though the radius at which this maximum is reached varies by a large factor). Examples of $H / R$ as a function of the disk radius are shown in Figure 17 for two choices of the BH spin, $a_{*}=0,0.998$. Notice how the two sets of curves agree very closely as far as their maxima are concerned. Therefore, regardless of the value of $a_{*}$, if we wish to have $(H / R)_{\max } \lesssim 0.1$, we require the dimensionless disk luminosity $l \equiv L / L_{\text {Edd }}$ to be $\lesssim 0.3$. 


\section{REFERENCES}

Abramowicz, M. A., Chen, X., Kato, S., Lasota, J.-P., \& Regev, O. 1995, ApJ, 438, L37

Abramowicz, M. A., Czerny, B., Lasota, J. P., \& Szuszkiewicz, E. 1988, ApJ, 332, 646

Abramowicz, M. A., \& Kluzńiak, W. 2001, A\&A, 374, L19

Afshordi, N., \& Paczyński, B. 2003, ApJ, 592, 354

Arnaud, K. A. 1996, in ASP Conf. Ser. 101, Astronomical Data Analysis Software and Systems V, ed. G. H. Jacoby \& J. Barnes (San Francisco: ASP), 17

Aschenbach, B. 2004, A\&A, 425, 1075

Belczynski, K. \& Bulik, T. 2002, ApJ, 574, L147

Belloni, T. 2004, Nuclear Physics B Proc. Suppl., 132, 337

Belloni, T., Klein-Wolt, M., Mendez, M., van der Klis, M., \& van Paradijs, J. 2000, A\&A, 355,271

Belloni, T., Mendez, M., King, A. R., van der Klis, M., \& van Paradijs, J. 1997, ApJ, 488, L109

Blandford, R. D., \& Znajek, R. L. 1977, MNRAS, 179, 433

Campanelli, M., Lousto, C. O., \& Zlochower, Y. 2006 (gr-qc/0604012)

Chapuis, C., \& Corbel, S. 2004, A\&A, 414, 659

Connors, P. A., Stark, R. F., \& Piran, T. 1980, ApJ, 235, 224

Davis, S. W., Blaes, O. M., Hubeny, I., \& Turner, N. J. 2005, ApJ, 621, 372 (D05)

Davis, S. W., Done, C., \& Blaes, O. M. 2006, ApJ, submitted (astro-ph/0602245, D06)

Davis, S. W. \& Hubeny, I. 2006, ApJS, in press (astro-ph/0602499)

Dhawan, V., Goss, W. M., \& Rodríguez, L. F. 2000a, ApJ, 540, 863

Dhawan, V., Mirabel, I. F., \& Rodríguez, L. F. 2000b, ApJ, 543, 373

Dovčiak, M., Karas, V., \& Matt, G. 2004, MNRAS, 355, 1005

Ebisawa, K., Ogawa, M., Aoki, T., Dotani, T., et al. 1994, PASJ, 46, 375 
Ebisawa, K., et al. 1998, in The Hot Universe (Proc. IAU Symp. \#188), eds. K. Koyama, S. Kitamoto, \& M. Itoh (Kluwer: Dordrecht) p392

Fender, R. P., Garrington, S. T., McKay, D. J., Muxlow, T. W. B., Pooley, G. G., Spencer, R. E, Stirling, A. M., \& Waltman, E. B. 1999, MNRAS, 304, 865

Feroci, M., Matt, G., Pooley, G., Costa, E., Tavani, M., \& Belloni, T. 1999, A\&A, 351, 985

Frank, J., King, A., Raine, D. J. 2002, Accretion Power in Astrophysics (Cambridge: Cambridge Univ. Press)

Gammie, C. F. 1999, ApJ, 522, L57

Gierlinśki, M., \& Done C. 2004, MNRAS, 347, 885

Gierlinśki, M., Maciolek-Niedzwiecki, A., \& Ebisawa, K. 2001, MNRAS, 325, 1253

Greiner, J., Cuby, J. G., \& McCaughrean, M. J. 2001, Nature, 414, 522

Harlaftis, E. T., \& Greiner, J. 2004, A\&A, 414, L13

Hawley, J. F., \& Balbus, Steven A. 2002, ApJ, 573, 738

Hua, X.-M., \& Titarchuk, L. 1995, ApJ, 449, 188

Jahoda, K., Markwardt, C. B., Radeva, Y., Rots, A., Stark, M. J., Swank, J. H., Strohmayer, T. E., \& Zhang, W. 2006, ApJS, in press (astro-ph/0511531)

Jonker, P. G., \& Nelemans, G. 2004, MNRAS, 354, 355

Kaiser, C. R., Gunn, K. F., Brocksopp, C., \& Sokoloski, J. L. 2004, ApJ, 612, 332

King, A. R., \& Kolb, U. 1999, MNRAS, 305, 654

Kirsch, M. G. F., Briel, U. G., Burrows, D., et al. 2005, SPIE, 5898, 22

Klein-Wolt, M., Fender, R. P., Pooley, G. G., Belloni, T., Migliari, S., Morgan, E. H., \& van der Klis, M. 2002, MNRAS, 331, 745

Kotani, T., Ebisawa, K., Dotani, T., Inoue, H., Nagase, F., Tanaka, Y., \& Ueda, Y. 2000, ApJ, 539, 413

Krolik, J. H. 1999, ApJ, 515, L73

Kubota, A., \& Done, C. 2004, MNRAS, 353, 980 
Lee, C.-H., Brown, G. E., \& Wijers, R. A. M. 2002, ApJ, 575, 996

Li, L.-X. 2003, Phys. Rev. D 67, 044007

Li, L.-X. 2004, PASJ, 56, 685

Li, L.-X., Zimmerman, E. R., Narayan, R., \& McClintock, J. E. 2005, ApJS, 157, 335

Lightman, A. P., \& Shapiro, S. L. 1976, ApJ, 203, 701

Lynden-Bell, D., \& Pringle, J. E. 1974, MNRAS, 168, 603

MacFadyen, A. I., \& Woosley, S. E. 1999, ApJ, 524, 262

Makishima, K., Maejima, Y., Mitsuda, K., Bradt, H. V., Remillard, R. A., Tuohy, I. R., Hoshi, R., \& Nakagawa, M. 1986, ApJ, 308, 635

Makishima, K., Tashiro, M., Ebisawa, K., Ezawa, H., Fukazawa, Y., Gunji, S., Hirayama, M., et al. 1996, PASJ, 48, 171

Martocchia, A., Matt, G., Karas, V., Belloni, T., \& Feroci, M. 2002, A\&A, 387, 215

Martocchia, A., Matt, G., Karas, V., Belloni, T., \& Feroci, M. 2004, Nuclear PhS, 132, 404

McClintock, J. E., \& Remillard, R. A. 2006, in Compact Stellar X-ray Sources, eds. W. H. G. Lewin \& M. van der Klis (Cambridge: Cambridge Univ. Press), 157 (MR06)

McKinney, J. C. \& Gammie, C. F. 2004, ApJ, 611, 977

Meier, D. L. 2003, New Astron. Rev., 47, 667

Merloni, A., Fabian, A. C., \& Ross, R. R. 2000, MNRAS, 313, 193

Middleton, M., Done, C., Gierlinśki, M., \& Davis, S. W. 2006, MNRAS, submitted (astro$\mathrm{ph} / 0601540)$

Miller, J. M., Fabian, A. C., Nowak, M. A., \& Lewin, W. H. G. 2004, to appear in the Proc. of the 10th Annual Marcel Grossman Meeting on General Relativity (astro-ph/0402101)

Miller-Jones, J. C. A., Rupen, M. P., Trushkin, S. A., Pooley, G. G., \& Fender , R. P. 2006, ATEL \#758

Mirabel, I. F., \& Rodríguez, L. F. 1994, Nature, 371, 46

Mirabel, I. F., \& Rodríguez, L. F. 1999, ARAA, 37, 409 
Mitsuda, K., Inoue, H., Koyama, K., et al. 1984, PASJ, 36, 741

Morgan, E. H., Remillard, R. A., \& Greiner, J. 1997, ApJ, 482, 993

Muno, M. P., Remillard, R. A., Waltman, E. B., et al. 2001, ApJ, 556, 515

Narayan, R., Kato, S., \& Honma, F. 1997, ApJ, 476, 49

Narayan, R., \& Yi, I. 1994, ApJ, 428, L13

Narayan, R., \& Yi, I. 1995, ApJ, 444, 231

Nityananda, R., \& Narayan, R. 1982, MNRAS, 201, 697

Novikov, I., \& Thorne, K. S. 1973, Astrophysics of Black Holes, in Black Holes, Gordoan \& Breach, NY

Park, S. Q., Miller, J. M., McClintock, J. E., Remillard, R. A., Orosz, J. A., Shrader, C. R., Hunstead, R. W., Campbell-Wilson, D., Ishwara-Chandra, C. H., Rao, A. P., \& Rupen, M. P. 2004, ApJ, 610, 378

Page, D. N., \& Thorne, K. S. 1974, ApJ, 191, 499

Podsiadlowski, Ph., Rappaport, S., \& Han, Z. 2003, MNRAS, 341, 385

Pringle, J. E., \& Rees, M. J. 1972, A\&A, 21, 1

Remillard, R. A., \& McClintock, J. E. 2006, ARAA, 44, in press (RM06)

Reynolds, C. S., \& Nowak, M. A. 2003, Physics Reports, 377, 389

Rodríguez, L. F., Gerard, E., Mirabel, I. F., Gómez, Y., \& Valźquez, A. 1995, ApJS, 101, 173

Rybicki, G. B., \& Lightman, A. P. 1979, Radiative Processes in Astrophysics (New York: John Wiley \& Sons)

Serlemitsos, P. J., Jalota, L., Soong, Y., et al. 1995, PASJ, 47, 105

Shafee, R., McClintock, J. E., Narayan, R., Davis, S. W., Li, L., \& Remillard, R. A. 2006, ApJ, 636, L113 (S06)

Shakura, N. I., \& Sunyaev, R. A. 1973, A\&A, 24, 337

Shapiro, S. L., \& Teukolsky, S. 1984, Black Holes, White Dwarfs and Neutron Stars (New York: John Wiley \& Sons) 
Shimura, T., \& Takahara, F. 1995, ApJ, 445, 780

Sobczak, G. J., McClintock, J. E., Remillard, R. A., Bailyn, C. D., \& Orosz, J. A. 1999, ApJ, 520, 776

Sobczak, G. J., McClintock, J. E., Remillard, R. A., Cui, W., Levine, A. M., Morgan, E. H., Orosz, J. A., \& Bailyn, C. D. 2000, ApJ, 544, 993

Swank, J. H. 1998, Nuclear Physics B Proc. Suppl., 69, 12

Tanaka, Y., \& Lewin, W. H. G. 1995, in X-ray Binaries, eds. W. Lewin, J. van Paradijs, \& E. van den Heuvel (Cambridge: Cambridge Univ. Press), p126

Thorne, K. S. 1974, ApJ, 191, 507

Titarchuk, L. 1994, ApJ, 434, 570

Toor, A., \& Seward, F. D. 1974, AJ, 79, 995

Török, G., Abramowicz, M. A., Kluzńiak, W., \& Stuchlk, Z. 2005, A\&A, 436, 1

Wald, R. M. 1984, General Relativity (Chicago: Univ. Chicago Press)

Willingale, R., Aschenbach, B., Griffiths, R. G., Sembay, S., Warwick, R. S., Becker, W., Abbey, A. F., \& Bonnet-Bidaud, J.-M. 2001, A\&A, 365, L212

Woosley, S. E. 1993, ApJ, 405, 273

Woosley, S. E., \& Heger, A. 2006, ApJ, 637, 914

Zhang, S. N., Cui, W., \& Chen, W. 1997, ApJ, 482, 155

Zombeck, M. V. 1990, Handbook of Space Astronomy \& Astrophysics, 2nd edition (Cambridge: Cambridge Univ. Press) 
Fig. 1.- Detailed results from fitting all 20 RXTE observations of GRS1915 in the thermal state with a model consisting of diskbb, a power-law, a Gaussian absorption line and a smedge component over the energy range $3-25 \mathrm{keV}$ (44 degrees of freedom). The data points circled in blue correspond to the crucial low-luminosity observations ( $\S 5, \S 6.1$, Appendix). The horizontal dashed line in the bottom panel is drawn at $\chi_{\nu}^{2}=1$. From top to bottom, the panels show: the ratio of the disk to total flux $f_{D}$, the two diskbb fitting parameters, the disk inner temperature $T_{\text {in }}(\mathrm{keV})$ and the normalization constant $K$, the disk flux $F_{D}$ $\left(10^{-7} \mathrm{erg} \mathrm{cm}^{-2} \mathrm{~s}^{-1}\right)$ and the power-law flux $F_{\mathrm{PL}}\left(10^{-8} \mathrm{erg} \mathrm{cm}^{-2} \mathrm{~s}^{-1}\right)$, the power-law photon index $\Gamma$, the central energy of the Gaussian absorption line $E_{\mathrm{Fe}}(\mathrm{keV})$, the intensity of the line $N_{\mathrm{Fe}}$ (photons $\mathrm{cm}^{-2} \mathrm{~s}^{-1}$ times 100) and the equivalent width of the line $\mathrm{EW}_{\mathrm{Fe}}(\mathrm{keV})$, the smedge edge energy $E_{S}(\mathrm{keV})$ and the smedge optical depth $\tau_{S}$, and finally the value of reduced chi-square. See $\S 4.1$ for further details.

Fig. 2.- Analogous to Figure 1, but with the non-relativistic disk model diskbb replaced by our relativistic disk model kerrbb2 (44 dof). The fits were done for a viscosity parameter $\alpha=0.01$. This figure is identical in structure with Figure 1 except that $T_{\text {in }}$ and $K$ are here replaced by two parameters of kerrbb2, namely, the $\mathrm{BH}$ spin parameter $a_{*}$ and the mass accretion rate $\dot{M}\left(10^{18} \mathrm{~g} \mathrm{~s}^{-1}\right)$.

Fig. 3.- This figure is identical to Figure 2, except that the fits were computed for a viscosity parameter $\alpha=0.1$.

Fig. 4.- Results of fitting the 20 RXTE observations of GRS1915 in the thermal state with a model consisting of kerrbb2, a thermal Comptonization component comptt, a Gaussian absorption line and a smedge component (43 dof). The panels are the same as in Figures 2 and 3 except that $F_{\mathrm{PL}}$ and $\Gamma$ are replaced by the flux in the comptt component $F_{C}$ and the optical depth of the Comptonizing corona $\tau_{C}$. See $\S 4.2 .2$ for other details.

Fig. 5.- Results of fitting the 20 RXTE observations of GRS1915 in the thermal state with a model consisting of kerrbb2, a cutoff power-law component expabs* power, a Gaussian absorption line and a smedge component (43 dof). See $§ 4.2 .3$ for other details.

Fig. 6.- Fit results for a model including a sharp absorption edge for the five observations in Figures $2-5$ that have $\chi_{\nu}^{2} \gtrsim 1.5$. The small black data points with error bars are identical to those plotted in Figure 2, and the results of including the sharp edge in the fits are plotted as open red circles. Note the pair of panels near the bottom displaying the parameters of the edge component.

Fig. 7. - Spin parameter $a_{*}$ versus the Eddington-scaled luminosity $L / L_{\text {Edd }}$ for all $22 R X T E$ and ASCA observations of GRS1915 in the thermal state for two values of the viscosity 
parameter $\alpha$. The tail emission is modeled as a simple power law. For reasons discussed in $\S 6.1$ and the Appendix, the results are most trustworthy for $L / L_{\text {Edd }} \lesssim 0.3$; this limit is indicated here and below by the vertical dotted line. Data in this regime consistently give a very high estimate of the spin parameter of GRS1915, $a_{*} \rightarrow 1$, independent of $\alpha$ or any other details.

Fig. 8. - Same as Figure 7 except that the tail emission is modeled as a Comptonized plasma and only the results for $\alpha=0.01$ are shown. Results are displayed for three values of $T_{0}$, the temperature of the seed photons.

Fig. 9.- Same as Figure 7 except that the tail emission is modeled as a cutoff PL, and $\alpha=0.01$ only. The results are shown for three values of the cutoff energy $E_{\mathrm{c}}$.

Fig. 10.- Direct comparison of the results displayed in Figures 7-9 for the three different tail models, for $\alpha=0.01$ only. Note how very similar the results are, which shows that the results are not sensitive to the details of the spectral model used to fit the high-energy tail component in the spectrum.

Fig. 11. - (a) Effects on the spin estimate of GRS1915 as a result of varying the distance $D$ to the source over the range $11.0-12.5 \mathrm{kpc}$. The mass of the $\mathrm{BH} M$ and the inclination $i$ are correlated with $D$, as explained in $\S 5.2$. The results for $D=9 \mathrm{kpc}$ and $D=10 \mathrm{kpc}$ are not shown for reasons that are given in $§ 5.2$. (b) Effects of varying the BH mass $M$ over its allowed range, keeping $D$ fixed at $11.0 \mathrm{kpc}$ and $i$ fixed at $66.0^{\circ}$.

Fig. 12. - The single spin estimate obtained by M06, which is here referred to a distance $D=11 \mathrm{kpc}$, is indicated by the three blue triangles that are connected by a dashed line. Our results, which are based on the comptt tail model for $T_{0}=2.0 \mathrm{keV}$ and $\alpha=0.01$, are shown as red circles (see Fig. 8).

Fig. 13. - Illustrates the effect of including the returning radiation in the model. The primary effect is to shift the estimated Eddington-scaled luminosities to higher values. There is very little effect on the estimates of $\mathrm{BH}$ spin $a_{*}$.

Fig. 14. - Illustrates the effect of including a nonzero torque at the inner edge of the disk.

Fig. 15.- The behavior of three dimensionless quantities that depend only on the BH spin parameter: (a) The radius of the ISCO in gravitational units, (b) the specific binding energy at the ISCO, and (c) the Keplerian orbital frequency at the ISCO. The filled data points correspond to nominal estimates of the spins of the four BHs (see Table 4): from left to right, LMC X-3 $\left(a_{*}=0.20\right)$, GRO J1655-40 ( $\left.a_{*}=0.70\right), 4 \mathrm{U} 1543-47\left(a_{*}=0.80\right)$, and GRS1915 $\left(a_{*}=0.99\right)$. The horizontal lines in each panel indicate the values of each of the 
three quantities in question that correspond to the following key values of spin: $a_{*}=0$ (short-dashed line), $a_{*}=1$ (long-dashed line), and $a_{*}=0.998$ (dotted line; Thorne 1974).

Fig. 16. - Ratio of disk thickness $H$ to radius $R$, plotted against $R / R_{\text {in }}$, for Eddingtonscaled values of luminosity $l=\dot{m}$ in steps of $\Delta l=0.1$ (from $l=0.1$ to $l=1.2$ upward). The results are for a Newtonian disk in which $R_{\text {in }}$ is the radius of the inner edge. The horizontal dashed line corresponds to $H / R=0.1$. (See Fig. 17 for the relativistic case.)

Fig. 17.- Ratio of disk thickness $H$ to radius $R$ for a relativistic disk around a Kerr black hole, plotted against $R / R_{g}$, for Eddington-scaled values of luminosity $l=\dot{m}$ in steps of $\Delta l=0.1$ (from $l=0.1$ to $l=1.2$ upward). The inner radius of the disk is at the innermost stable circular orbit. The thick lines correspond to a non-rotating black hole $\left(a_{*}=0\right)$ and the thin lines to a maximally rotating black hole $\left(a_{*}=0.998\right)$. The horizontal dashed line corresponds to $H / R=0.1$. It is anticipated that the disk spectral models employed in this paper (diskbb, kerrbb2, bhspec) are most reliable when $H / R \lesssim 0.1$, which corresponds to the luminosity limit $l \lesssim 0.3$. (See Fig. 16 for the Newtonian case.)

Fig. 18.- (a) The allowed values of BH mass and distance for GRS1915 fall within the triangular region indicated (see text). (b) A summary of model-dependent distance estimates for GRS1915. The two relatively precise and disparate estimates at the bottom of the figure require comment: The one labeled "Radio/IR: lobes" is based on identifying a pair of extended IRAS sources as the regions where the jets of GRS1915 impact the ISM (Kaiser et al. 2004). The other estimate labeled "Near-IR systemic velocity $+21 \mathrm{~cm}$ " is based on a systemic velocity of $\gamma=-3 \pm 10 \mathrm{~km} \mathrm{~s}^{-1}$ and the Galactic rotation curve (Greiner et al. 2001). This latter estimate ignores the potentially sizable and unknown uncertainty associated with a possible peculiar component of radial velocity as well as any kick velocity that may have been imparted to the system during the formation of the BH (e.g., Jonker \& Nelemans 2004). References: (1) Rodríguez et al. 1995; (2) Dhawan et al. 2000a; (3) Dhawan et al. 2000b; (4) Chapuis \& Corbel 2004; (5) Kaiser et al. 2004; (6) Greiner et al. 2001. 


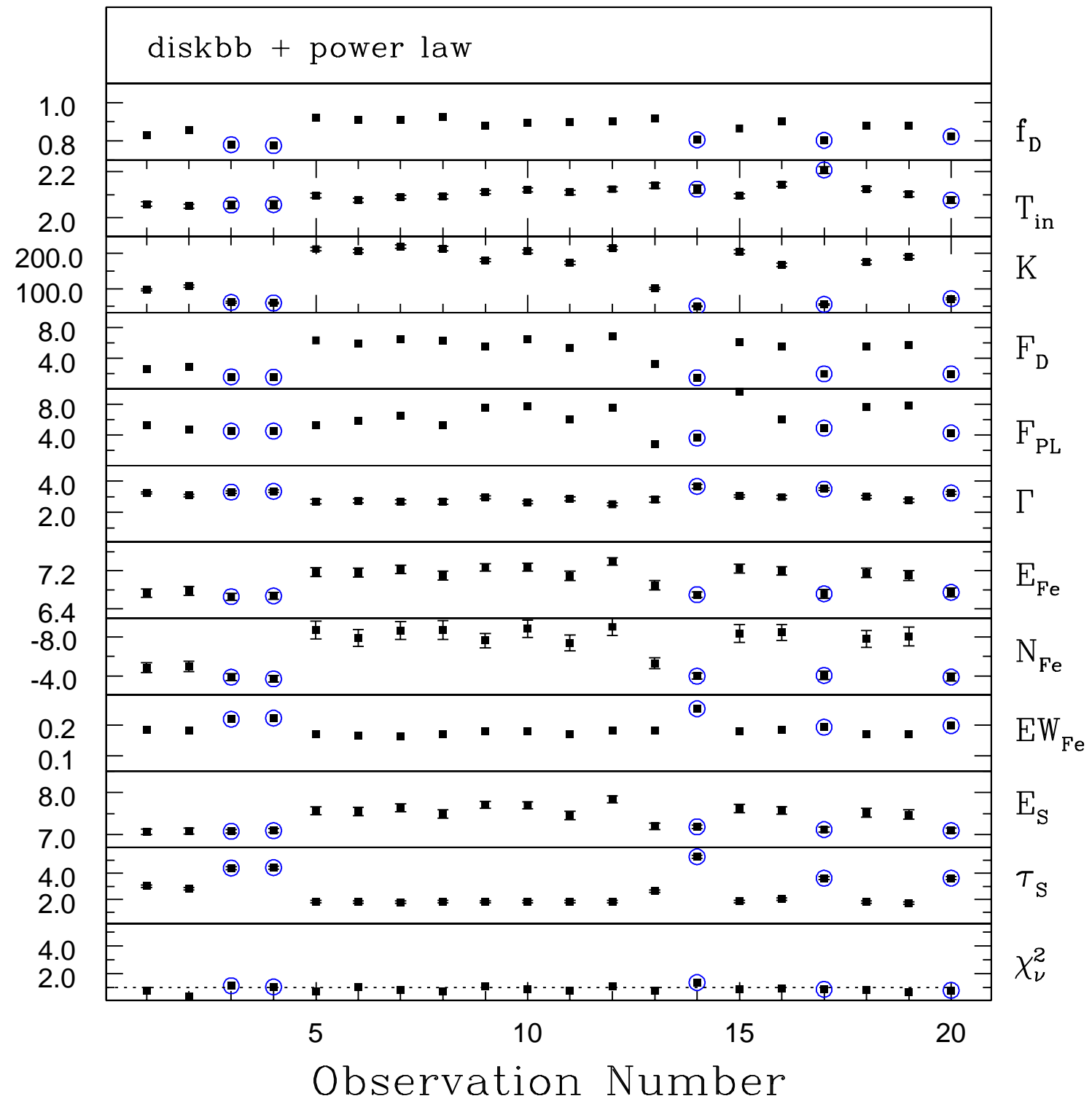

Fig. 1.- 


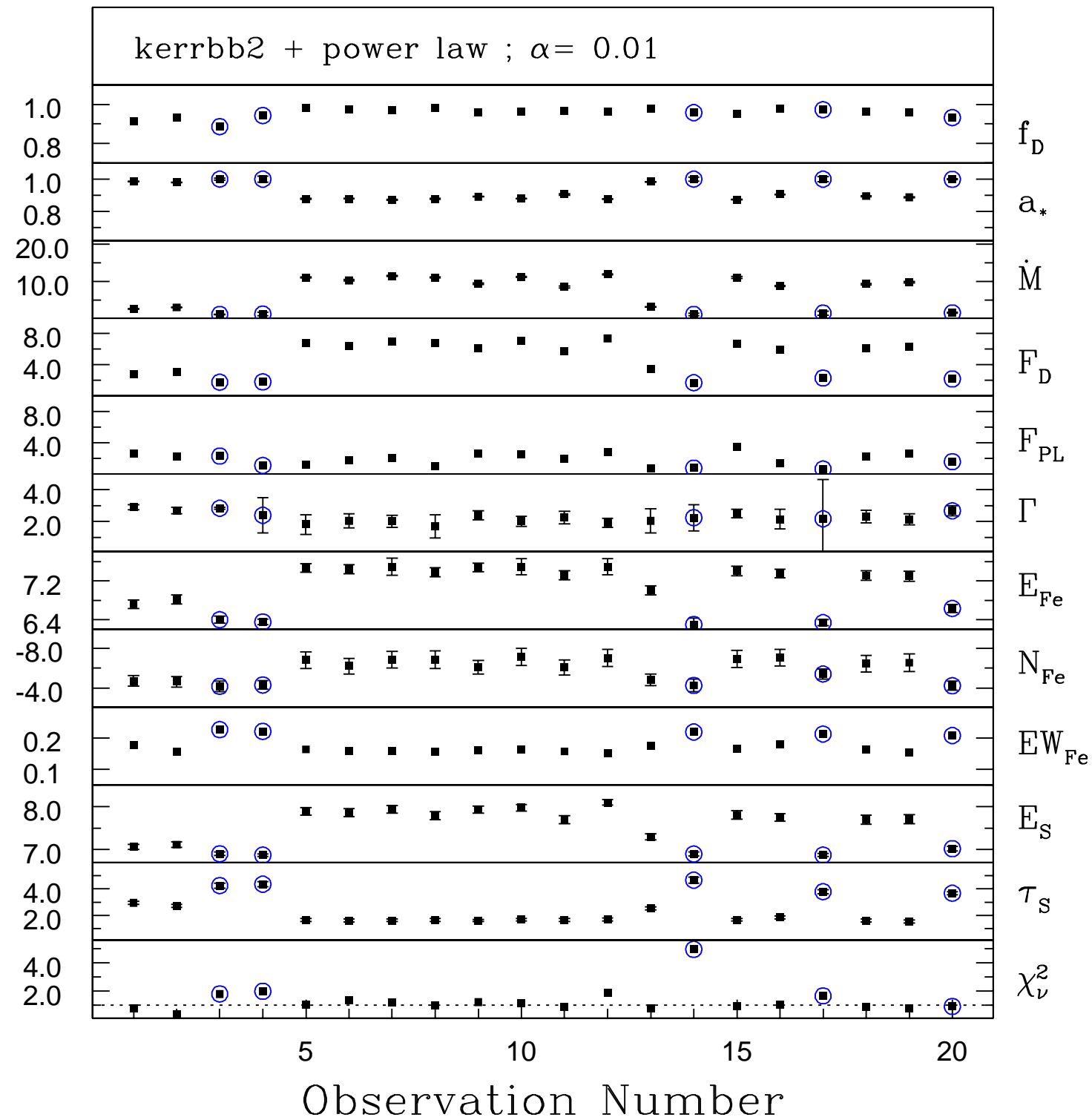

Fig. 2.- 


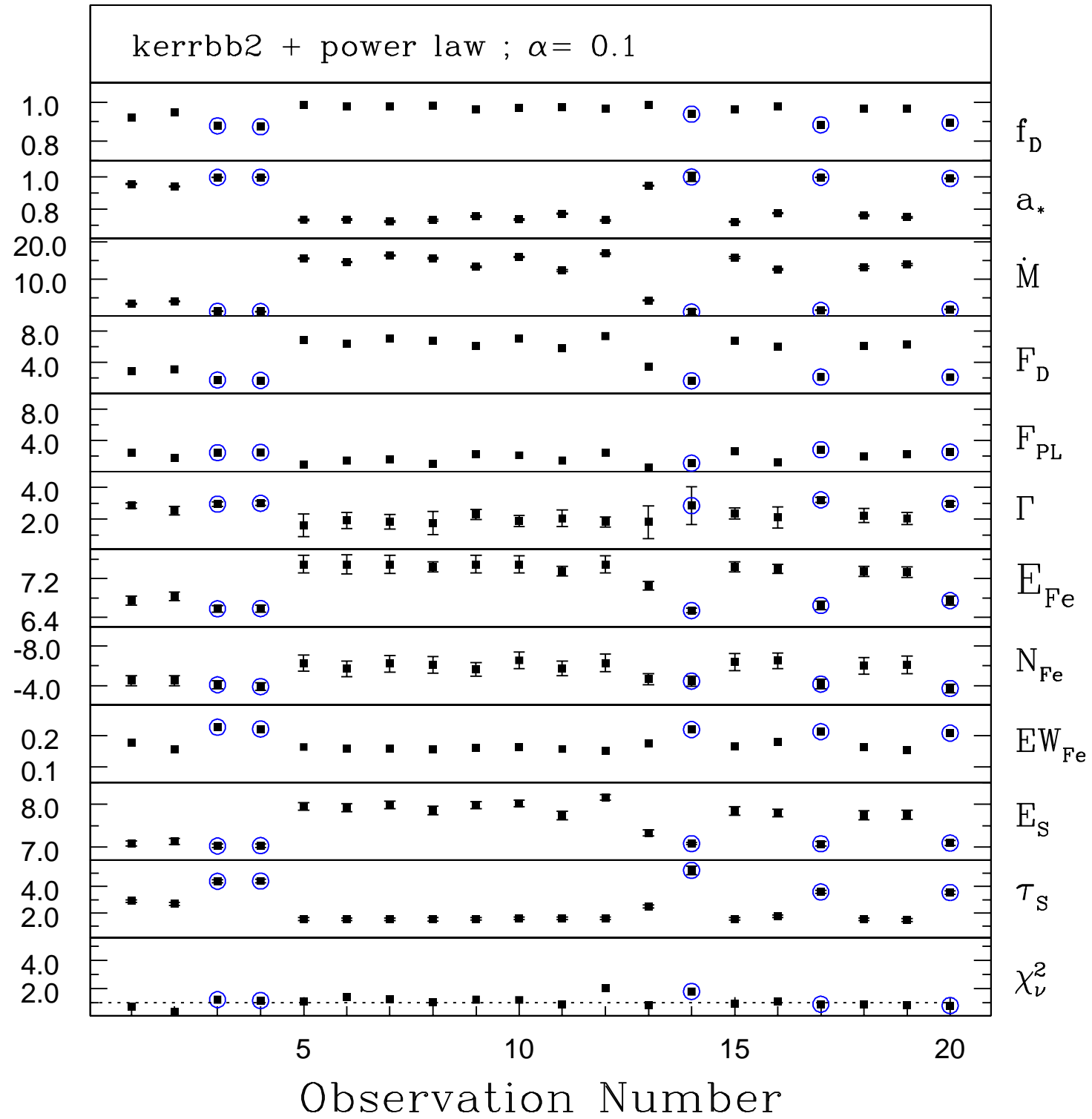

Fig. 3.- 


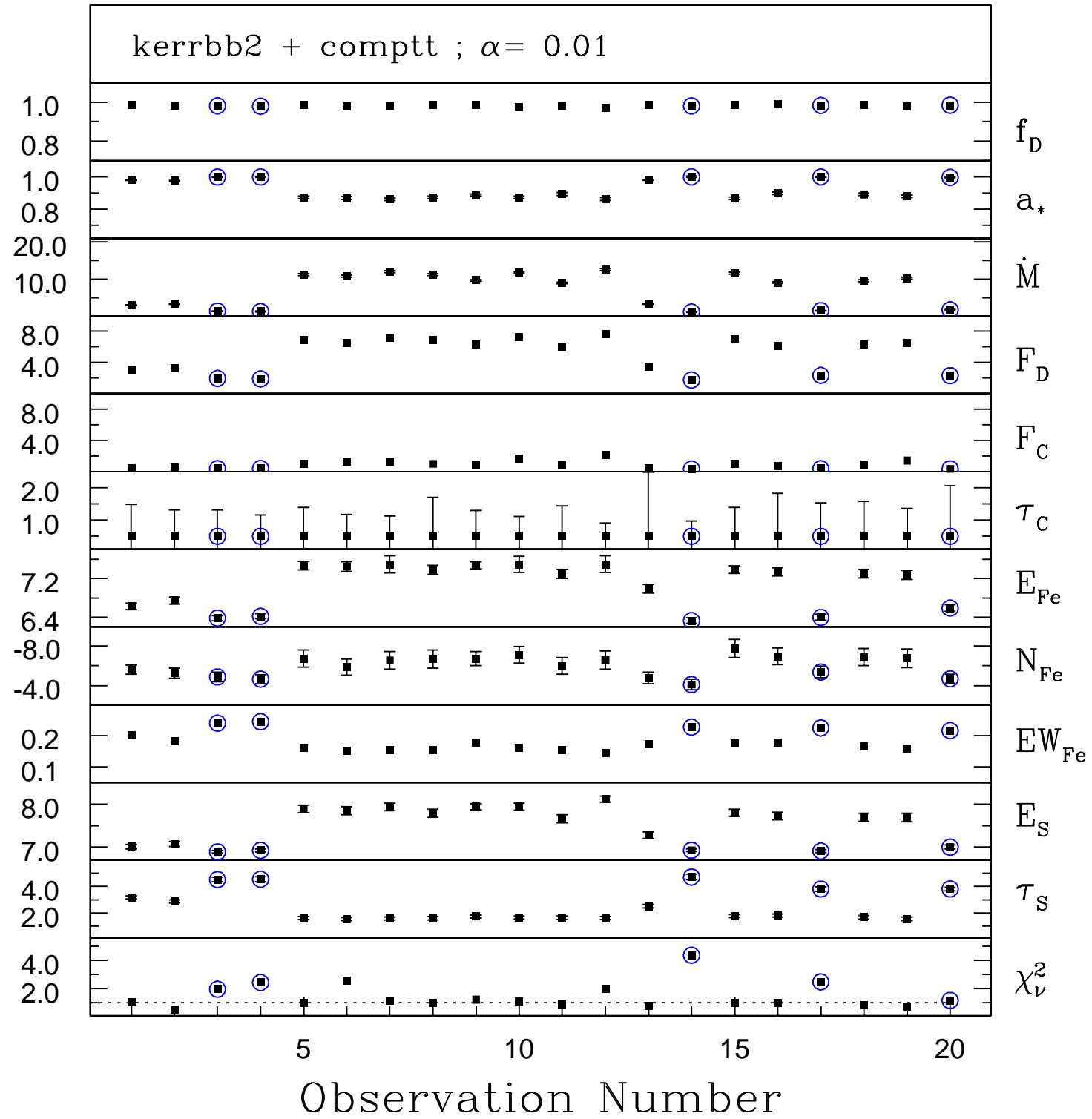

Fig. 4.- 


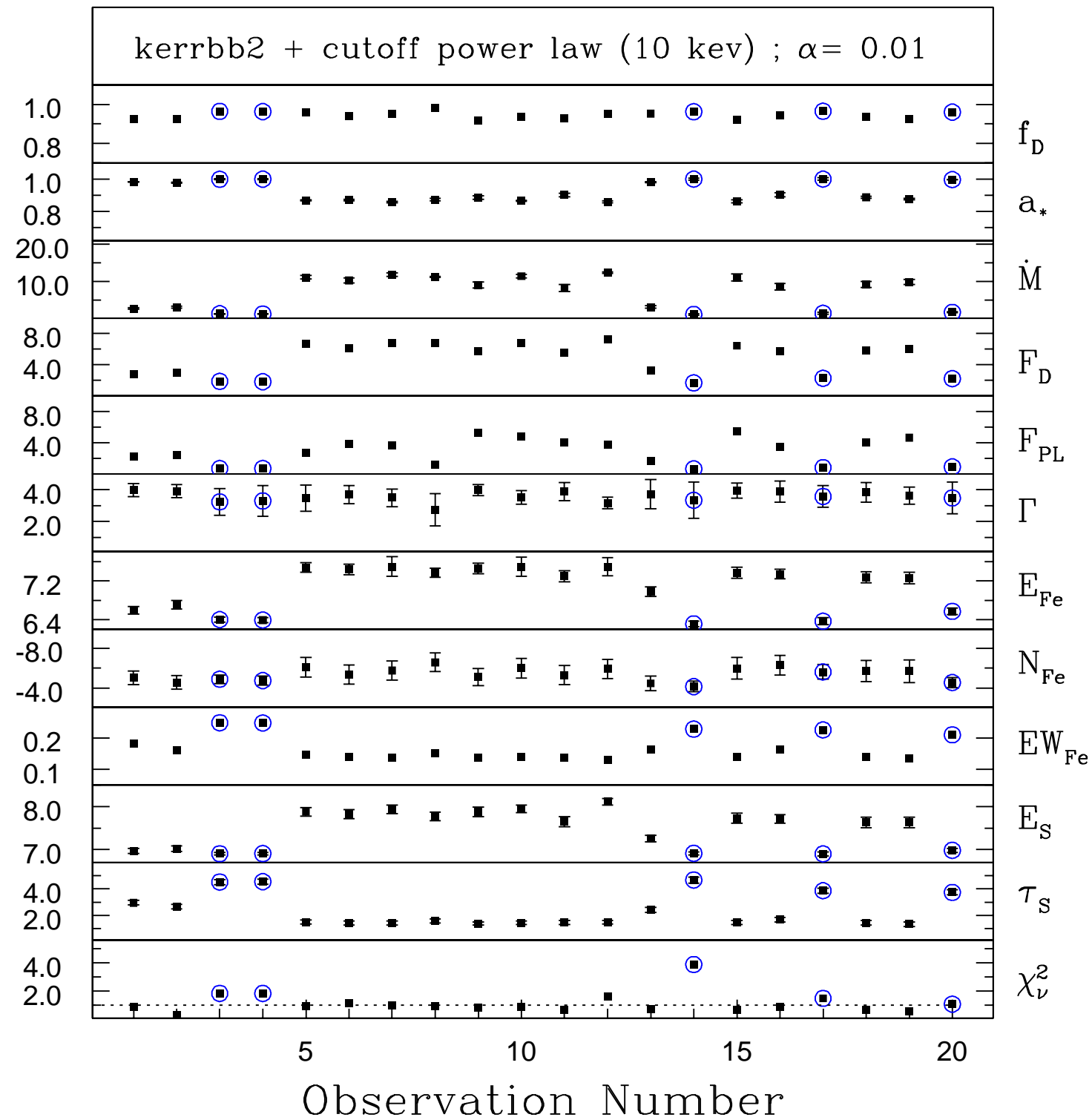

Fig. 5.- 


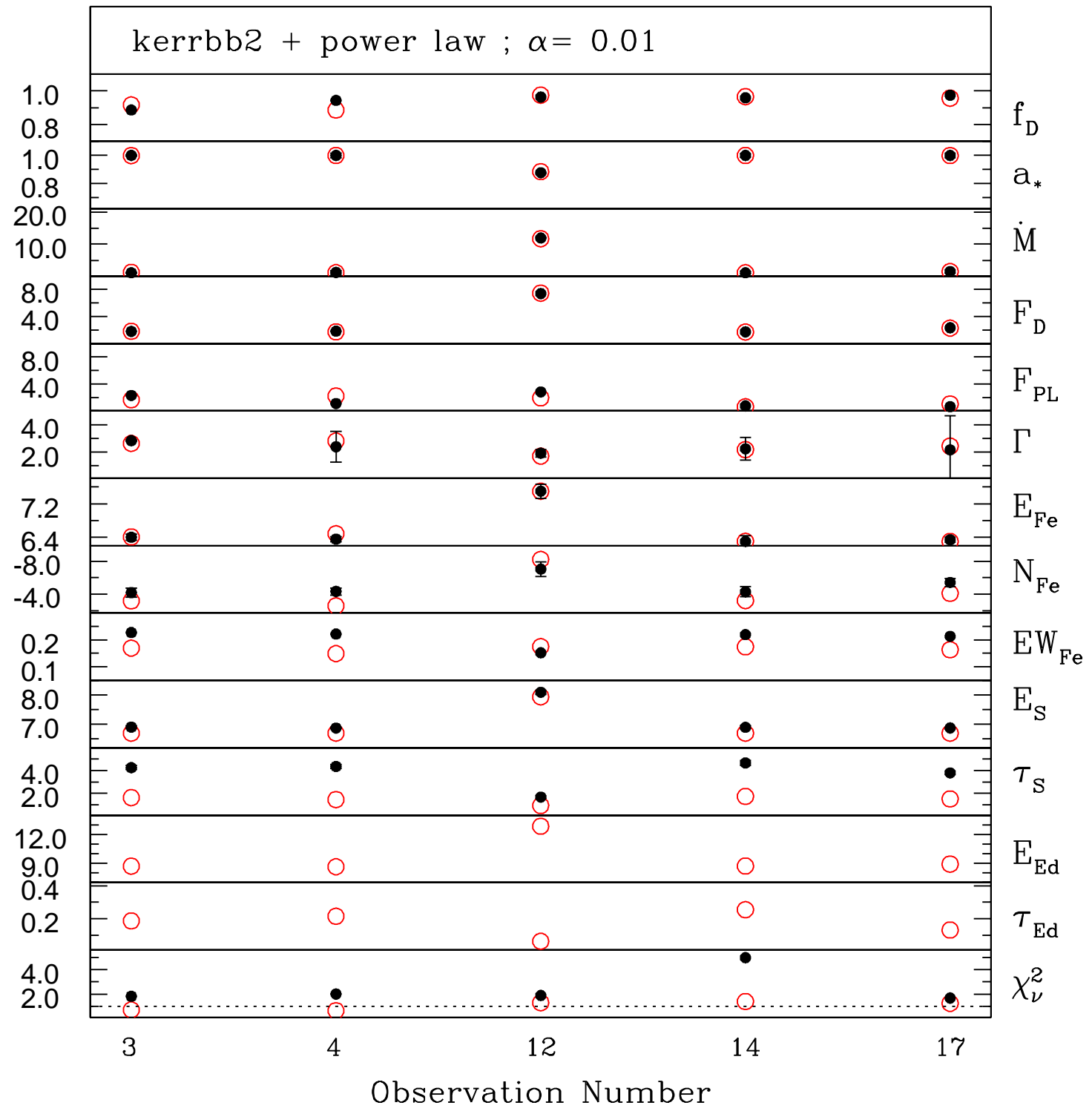

Fig. 6.- 


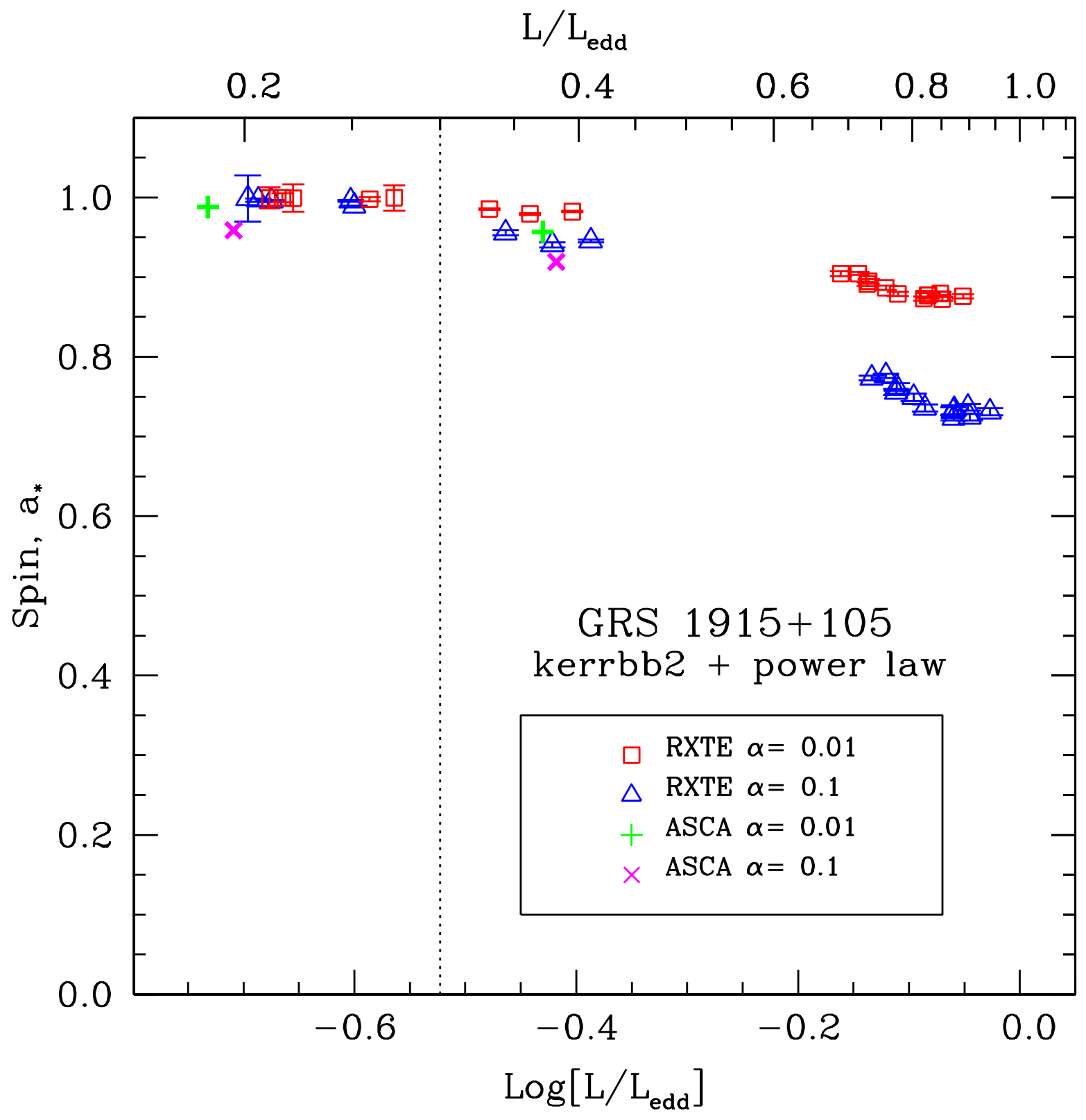

Fig. 7.- 


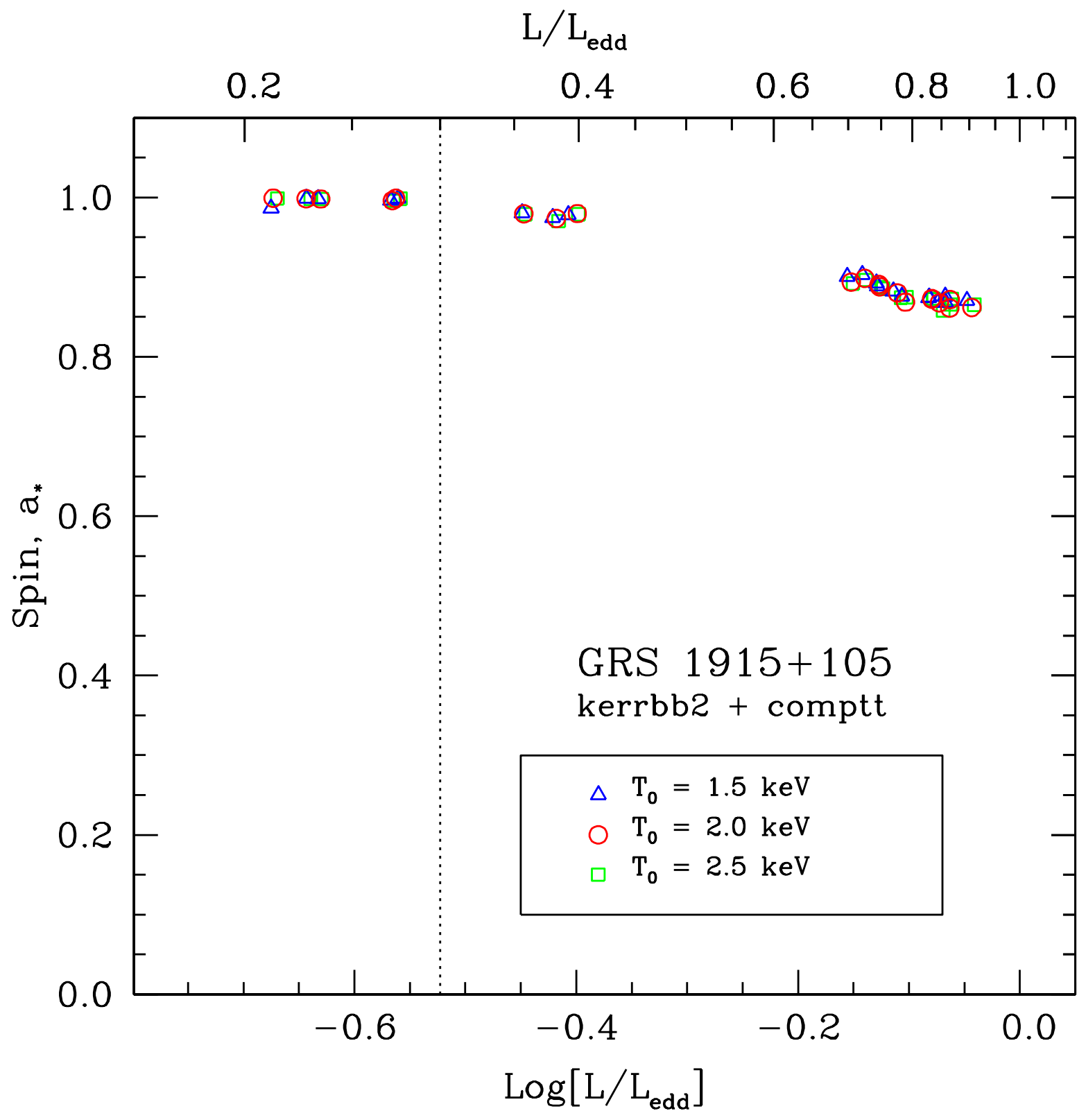

Fig. 8.- 


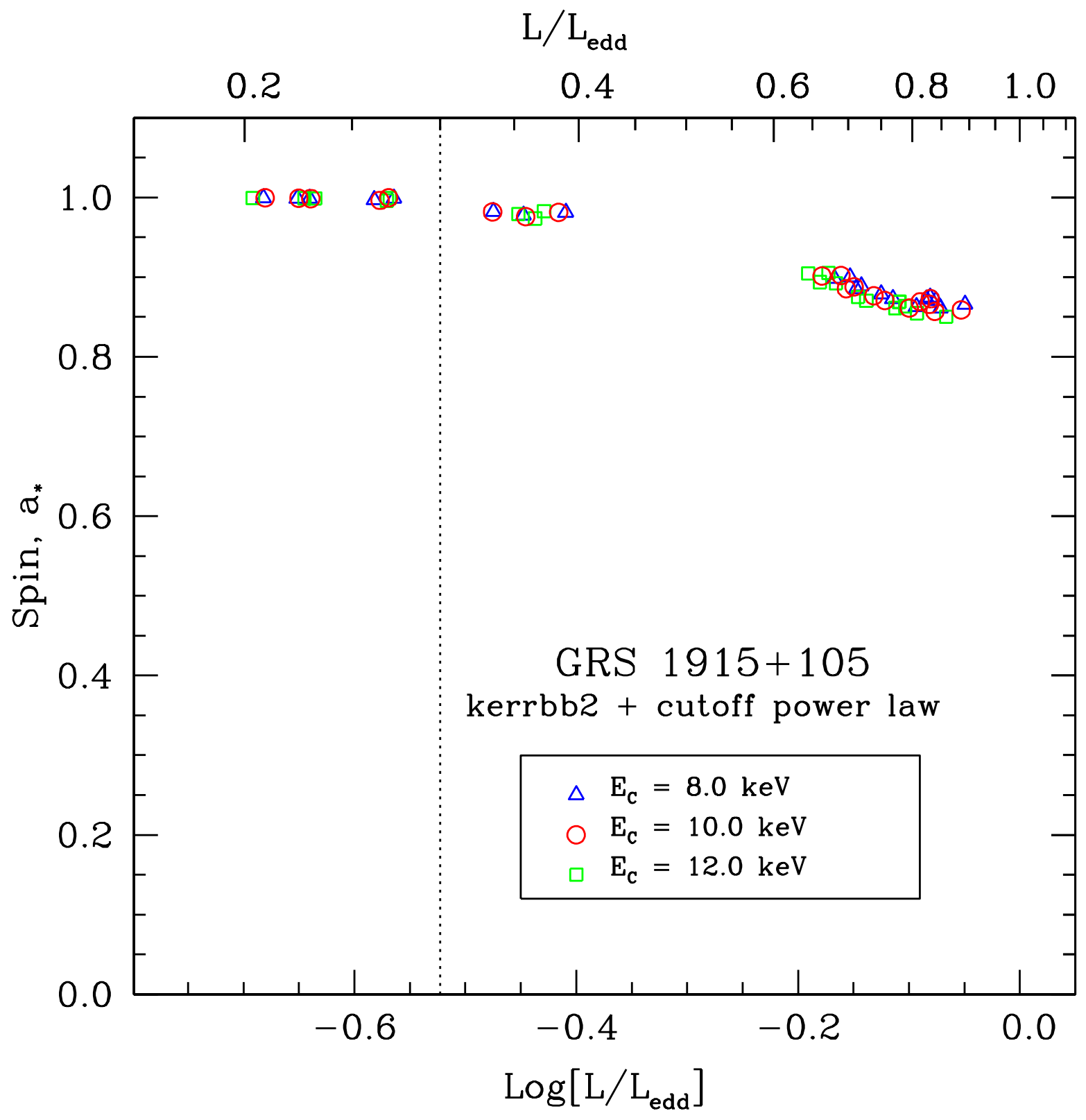

Fig. 9.- 


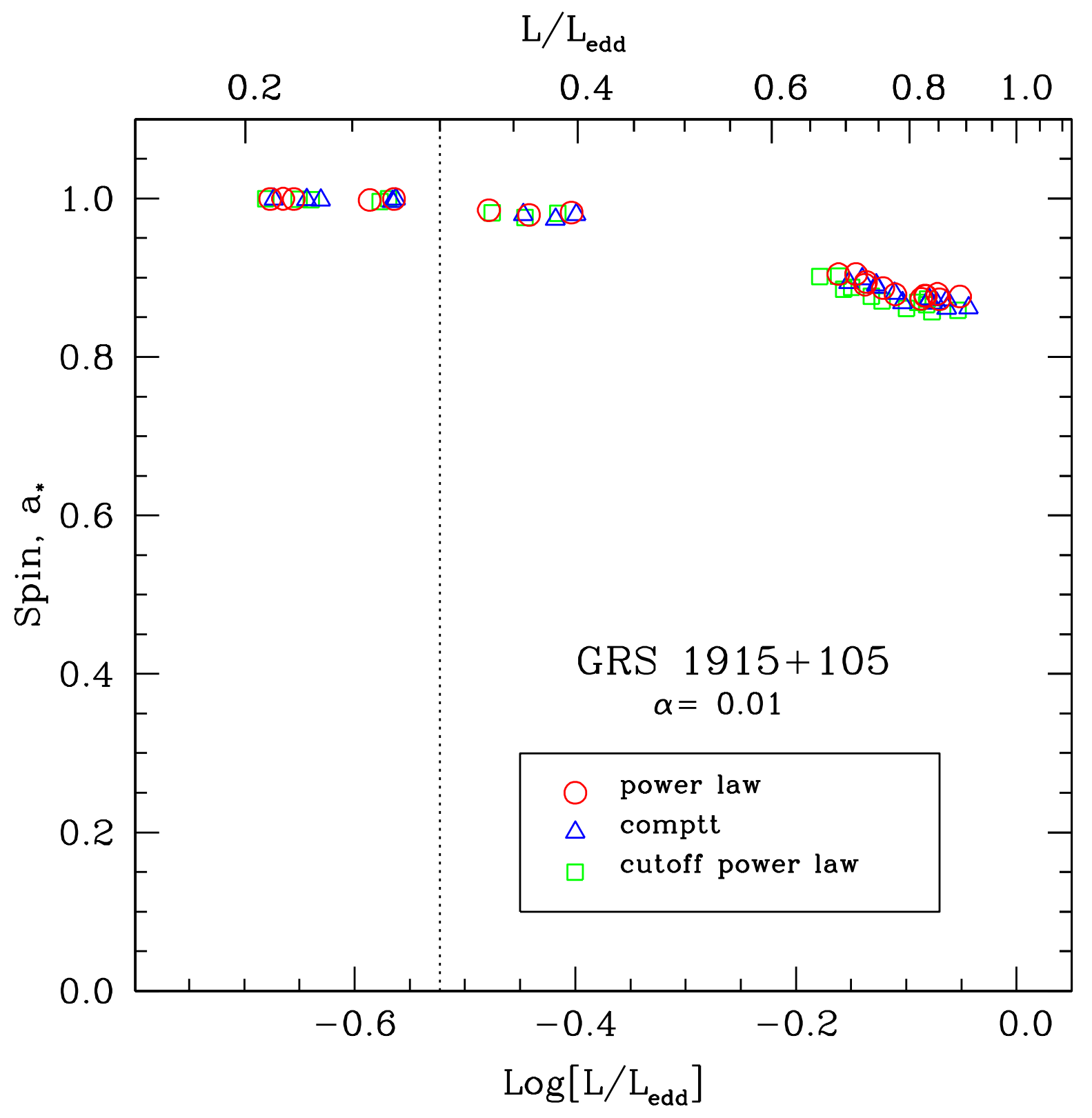

Fig. 10.- 

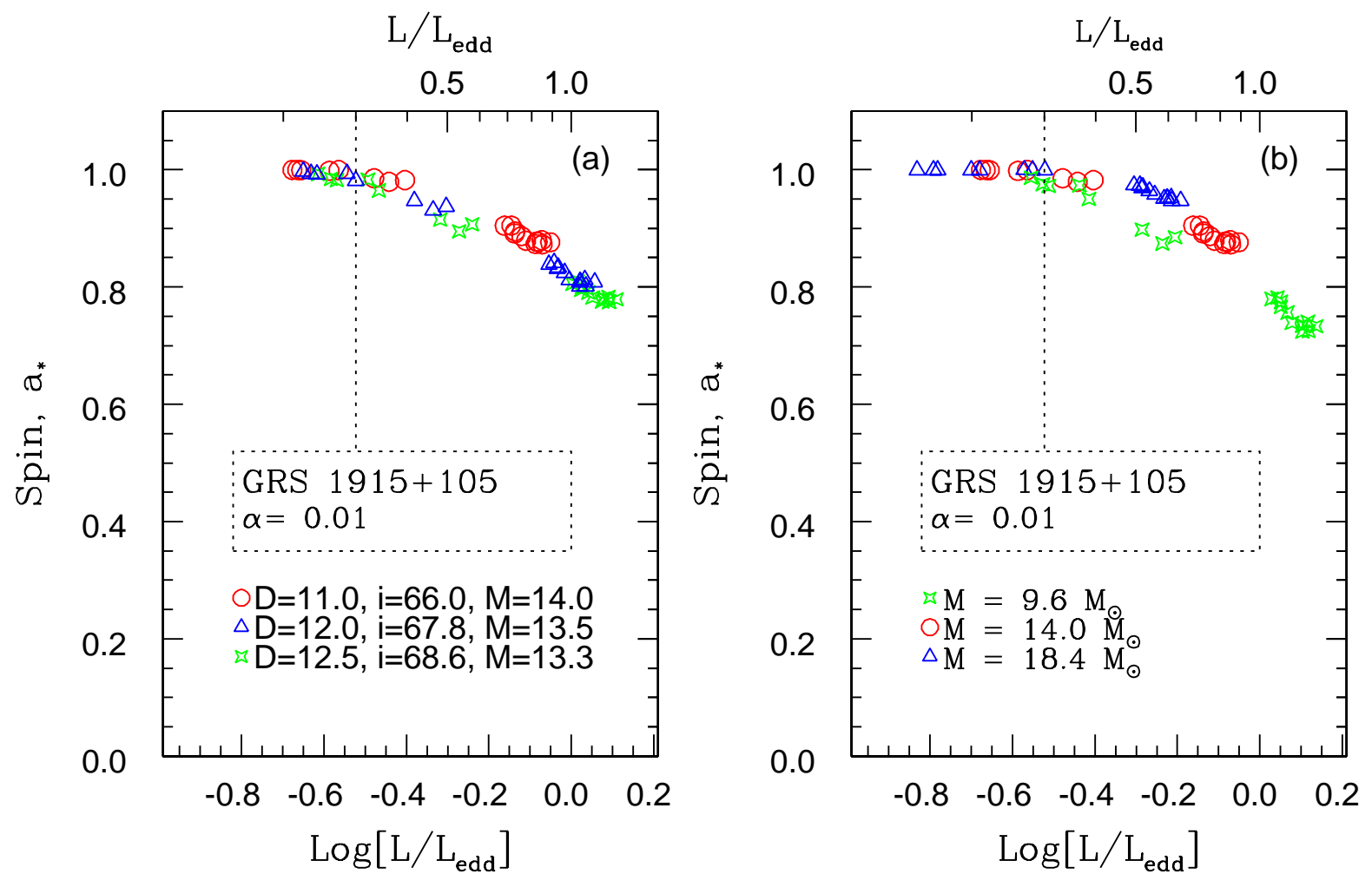

Fig. 11.- 


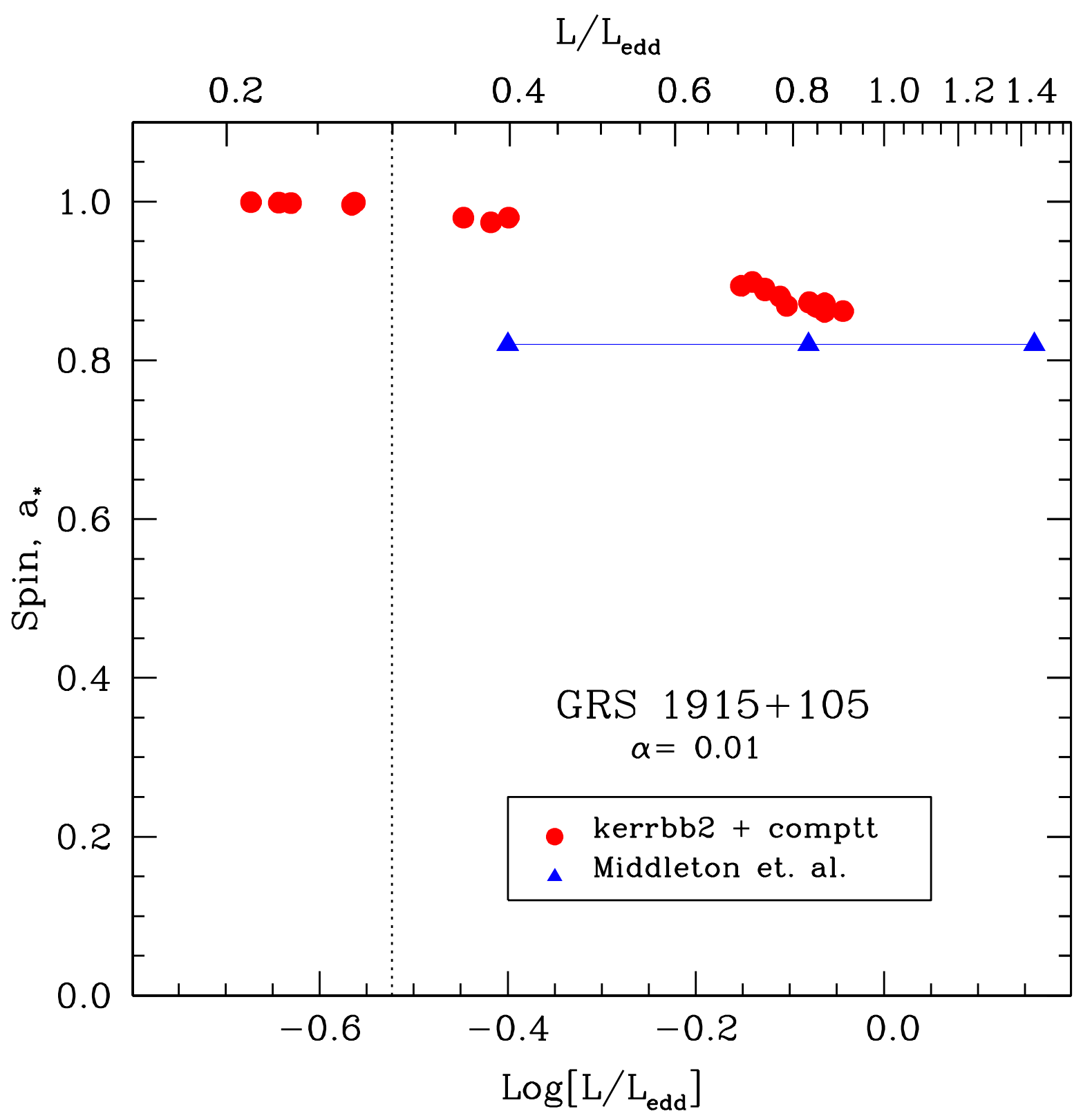

Fig. 12.- 


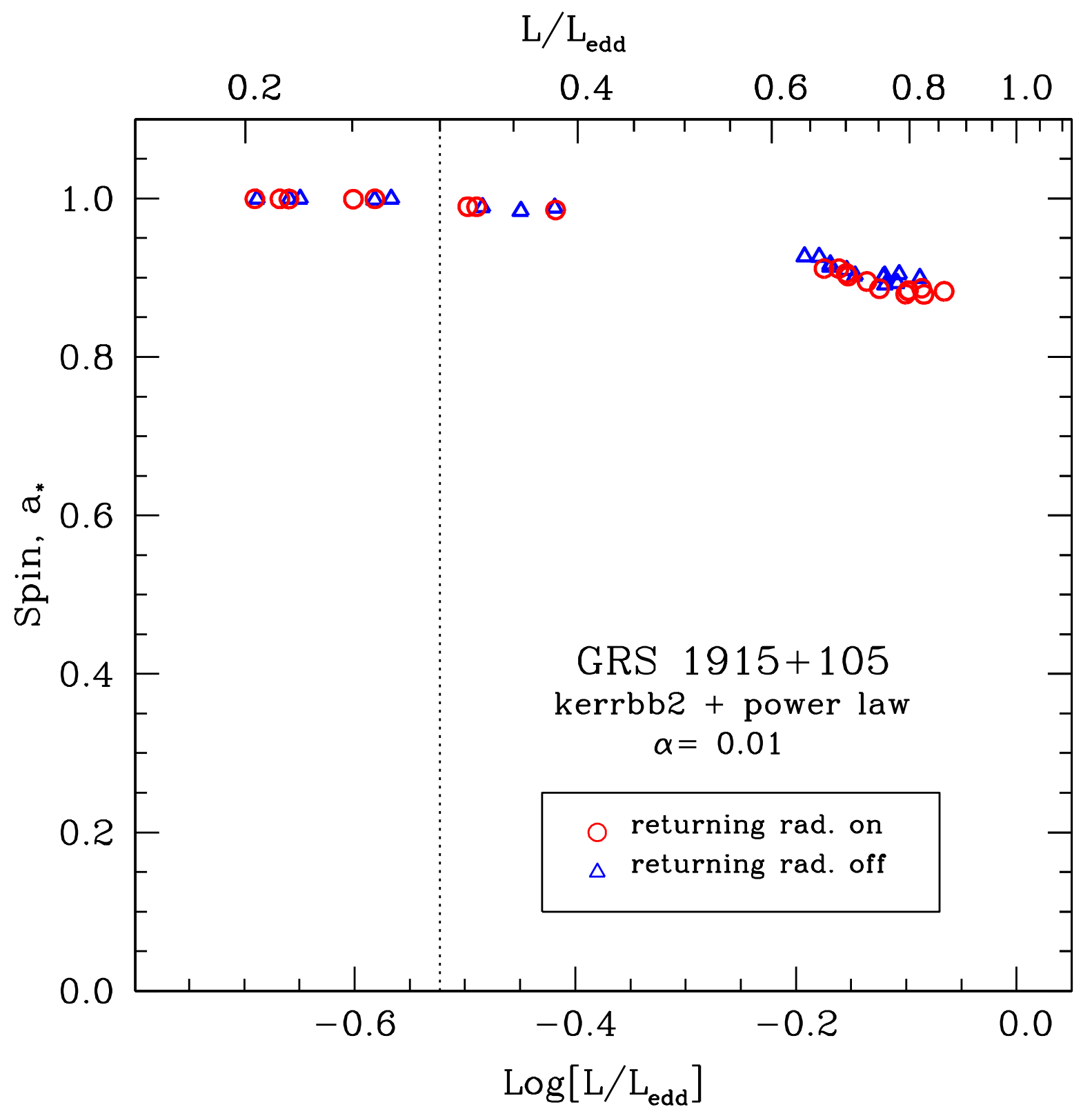

Fig. 13.- 


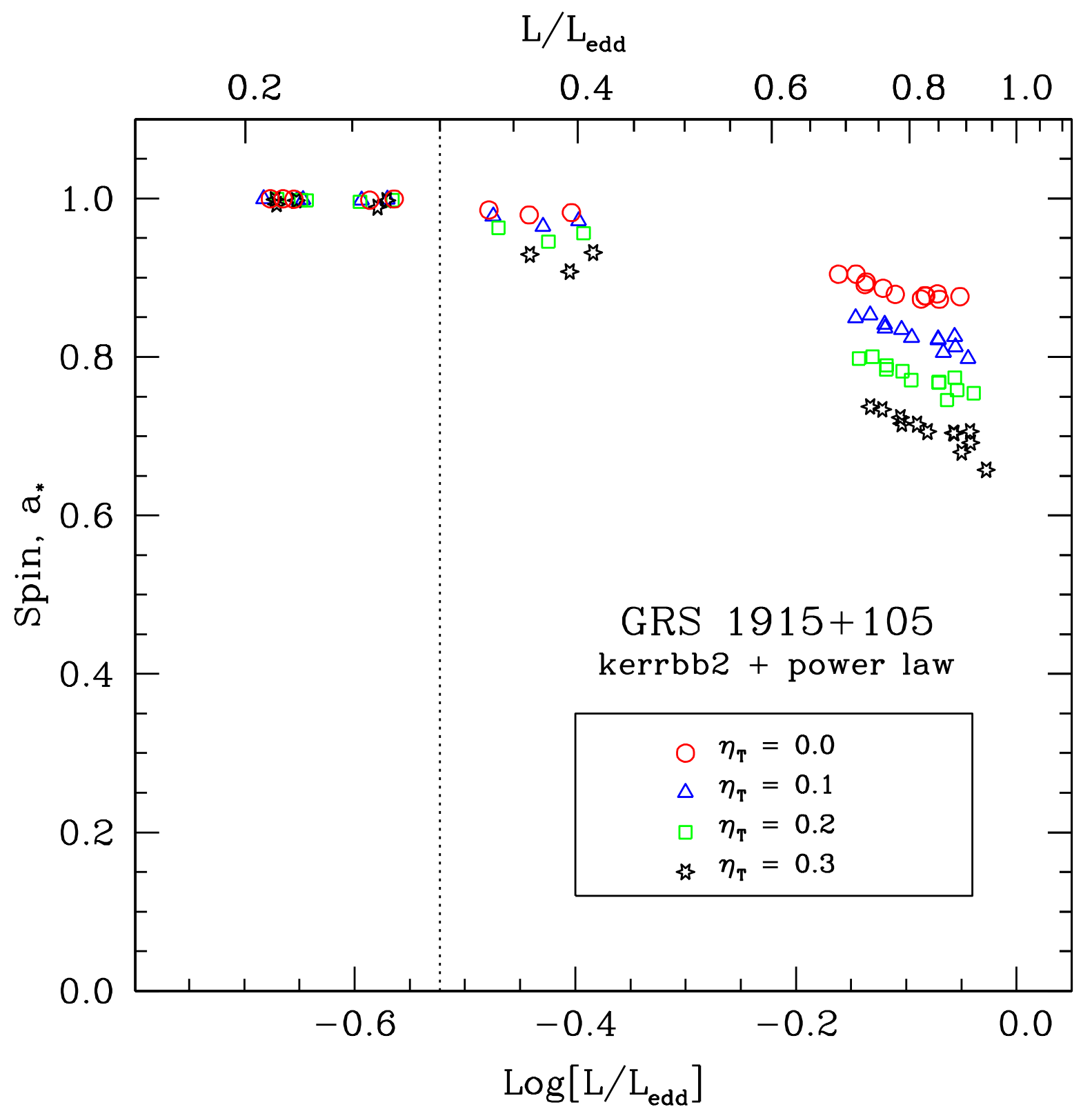

Fig. 14.- 


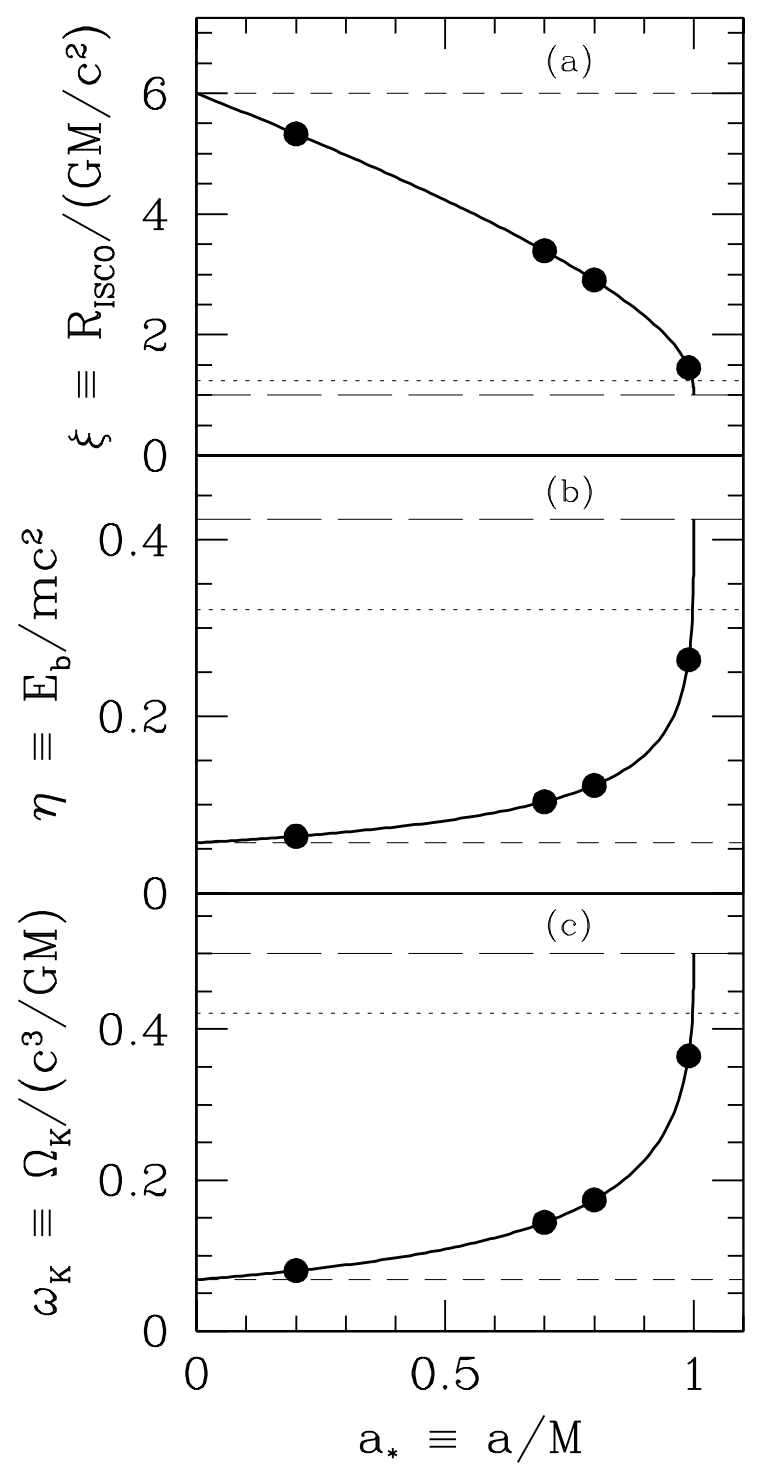

Fig. 15.- 


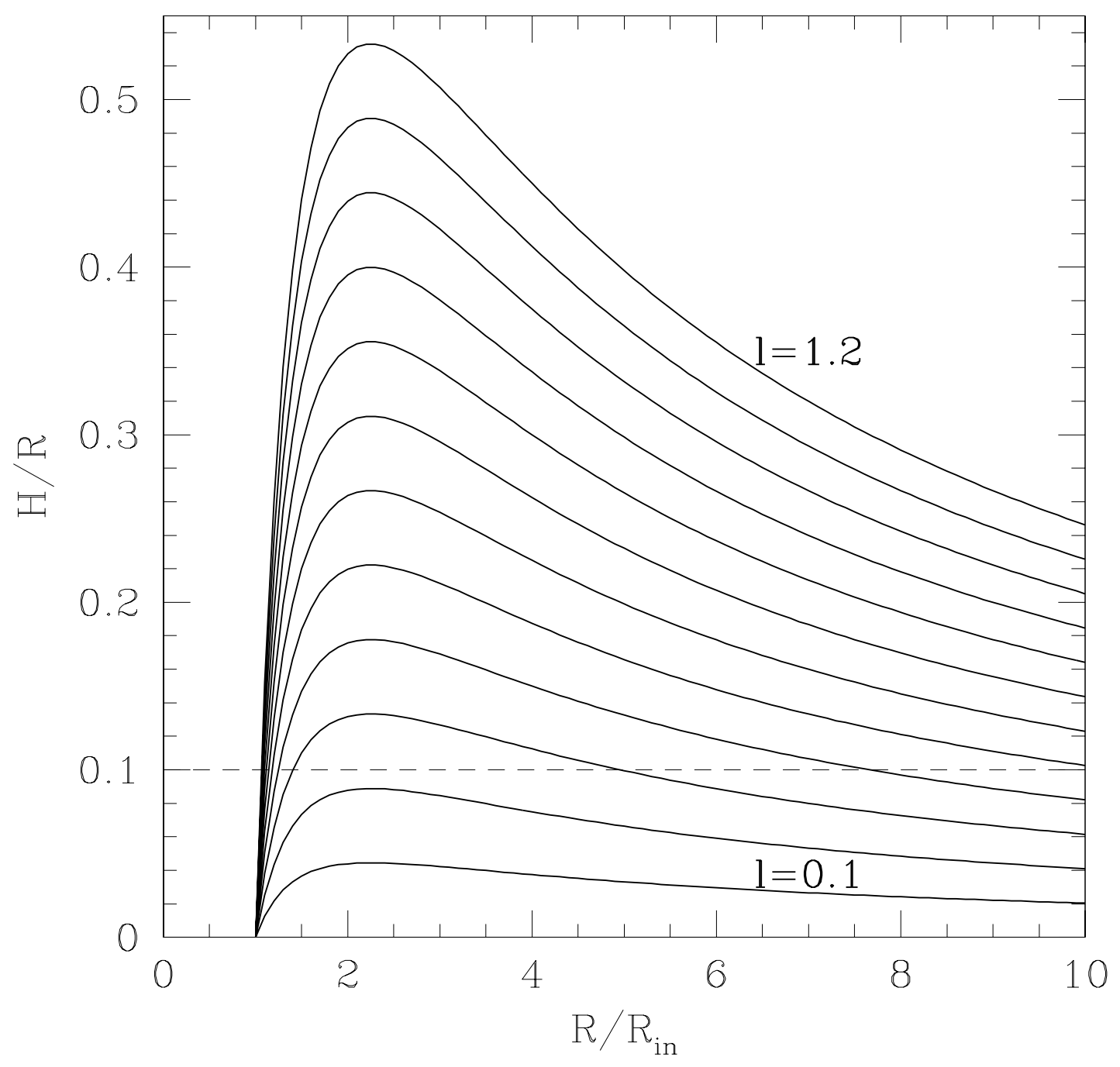

Fig. 16.- 


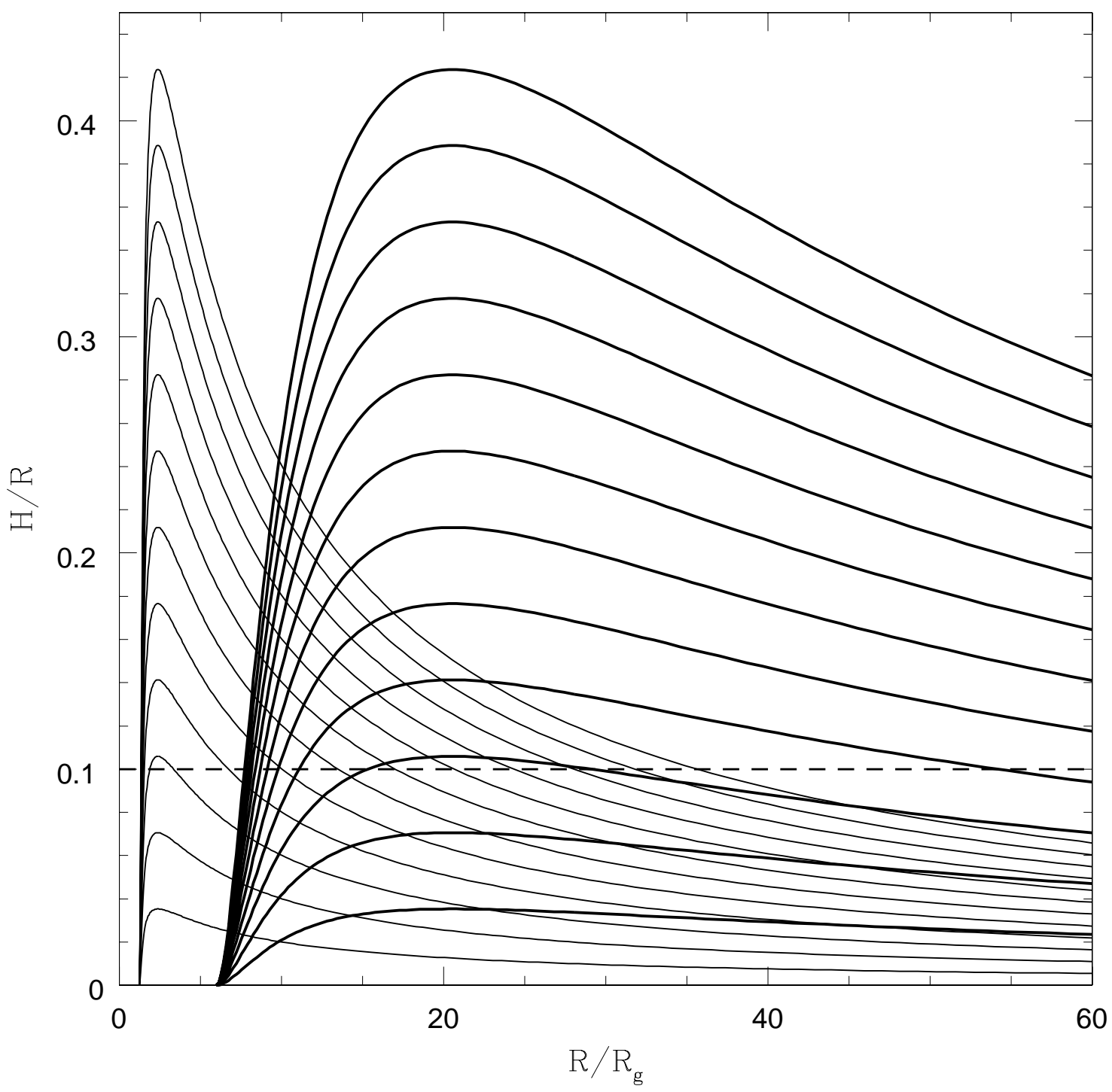

Fig. 17.- 


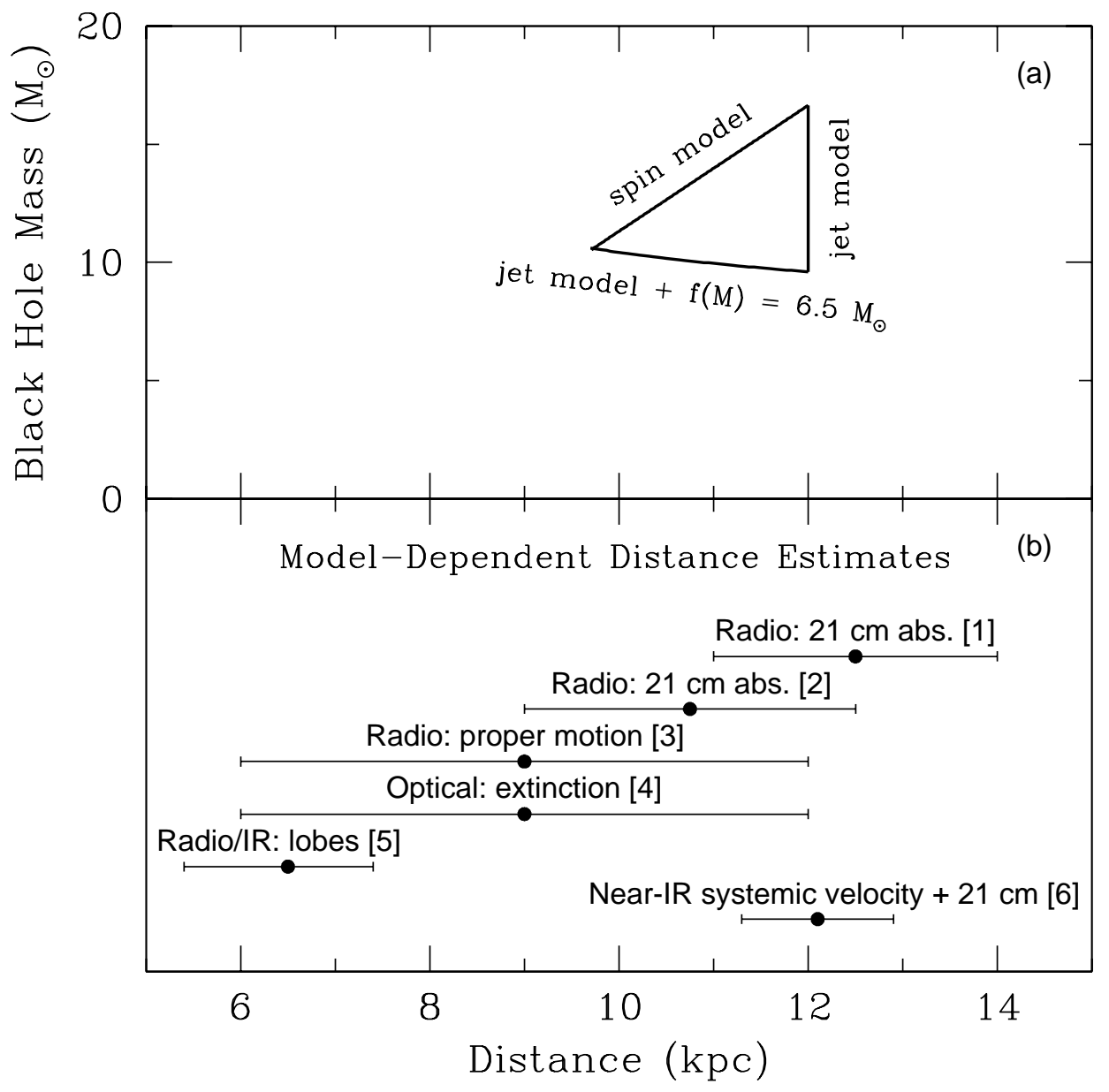

Fig. 18.- 